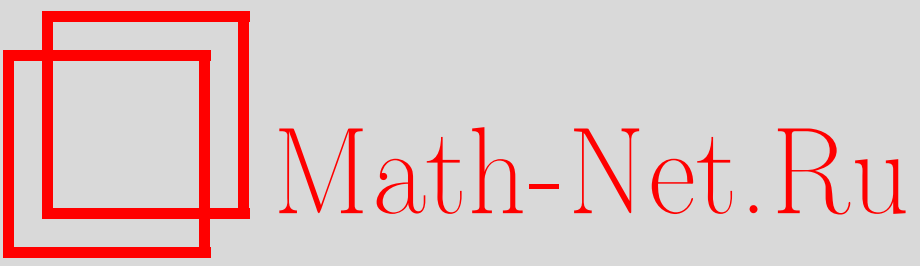

М. О. Корпусов, А. Г. Свешников, О разрушении решений класса сильно нелинейных волновых диссипативных уравнений типа Соболева с источниками, Изв. РАН. Сер. матем., 2005, том 69, выпуск 4, 89-128

DOI: https://doi.org/10.4213/im649

Использование Общероссийского математического портала Math-Net.Ru подразумевает, что вы прочитали и согласны с пользовательским соглашением

http: //www.mathnet.ru/rus/agreement

Параметры загрузки:

IP : 54.196 .121 .252

26 апреля 2023 г., 14:56:43 


\author{
М. О. Корпусов, А.Г. Свешников
}

\title{
О разрушении решений класса сильно нелинейных волновых диссипативных уравнений типа Соболева с источниками
}

\begin{abstract}
Рассмотрена абстрактная задача Коши для обыкновенного дифференциального уравнения первого порядка с нелинейными операторными коэффициентами. В качестве приложений приведены примеры сильно нелинейных волновых диссипативных уравнений типа Соболева. Для данной задачи получены достаточные, близкие к необходимым, условия как глобальной разрешимости, так и разрушения за конечное время. В частности, при дополнительных условиях на нелинейные операторы доказана разрешимость задачи в любом конечном цилиндре, кроме того, доказано разрушение решения задачи за конечное время при некоторых условиях, налагаемых на норму начальной функции. Приведены задачи для уравнений типа Соболева, удовлетворяющие введенным условиям.

Библиография: 33 наименования.
\end{abstract}

\section{$\S 1$. Введение. Постановка задачи}

Целью настоящего исследования является получение оптимальных результатов типа теорем “существования-несушествования" решения начальных краевых задач для класса сильно нелинейных волновых диссипативных уравнений типа Соболева, которые в абстрактной постановке являются задачей Коши для уравнений с операторными коэффищиентами в банаховых пространствах

$$
\frac{d}{d t}\left(\mathbb{A}_{0} u+\sum_{j=1}^{N} \mathbb{A}_{j}(u)\right)+\mathbb{L} u+\mathbb{D P}(u)=\mathbb{F}(u), \quad u(0)=u_{0},
$$

и оценок снизу и сверху времени разрушения решений задачи (1.1).

Под оптимальными результатами мы понимаем такие, что при одних исходных параметрах задачи имеет место разрушение решений, а при других - глобальная разрешимость. В этой связи мы намеренно сузили класс рассматриваемых уравнений типа Соболева до такого класса, для которого можно получить оптимальные результаты. Кроме того, мы рассматриваем лишь такие задачи, для которых может быть доказана единственность решения, понимаемого в том или ином смысле.

Работа выполнена при финансовой поддержке РФФИ (проекты № 02-01-00253, 02-0106038)

(C) М.О. КорпУСов, А.Г. Свешников, 2005 
В последнее время в математической физике стали интенсивно появляться новые сильно нелинейные волновые диссипативные уравнения псевдопараболического типа [1], [2], причем модельные уравнения оказываются либо третьего, либо пятого порядка с производной по времени первого порядка.

Исследованию уравнений псевдопараболического и соболевского типов посвяшено большое количество работ. Прежде всего отметим классическую работу [3] (последуюшие результаты по исследованию линейных уравнений типа Соболева см. в работах [4], [5]). Отметим также работу [6], где был предложен операторный метод исследования линейных уравнений типа Соболева высокого порядка. В работе [7] был предложен метод полугрупп с нетривиальными ядрами, который был успешно применен к исследованию разнообразных задач для нелинейных псевдопараболических задач.

Исследованию вопросов разрушения решений квазилинейных диссипативных "не волновых" уравнений с источниками посвящена классическая работа [8]. В этой работе был предложен оригинальный энергетический метод исследования разрушения решений уравнений формально параболического вида

$$
\mathbb{P} u_{t}=-\mathbb{A} u+\mathbb{F}(u)
$$

где существенно использовалась линейность и позитивность операторов $\mathbb{P}$ и $\mathbb{A}$, определенных на некотором гильбертовом пространстве. Далее в работе [9] данный подход был развит и применен для исследования, например, такого нелинейного уравнения:

$$
\left|u^{\prime}\right|^{q} u^{\prime}+\mathbb{A} u=\mathbb{F}(u, t) .
$$

Однако исследование случая производной по времени нелинейного эллиптического оператора осталось вне рамок предлагаемой в [8] техники.

В работе [10] методом нижних и верхних решений был исследован вопрос о разрушении решения и о глобальной разрешимости для уравнения типа Буссинеска с источником.

В работе [11] была предложена некоторая модификация энергетического метода Г. А. Левина [8], [9] и удалось рассмотреть случай сильно нелинейных псевдопараболических “не волновых" уравнений с источниками. В работах [12], [13] авторами были рассмотрены следующие операторно-дифференциальные уравнения:

$$
\begin{array}{rlrl}
\frac{d}{d t}\left(\mathbb{A}_{0} u+\sum_{j=1}^{N} \mathbb{A}_{j}(u)\right) & =\mathbb{F}(u), & u(0)=u_{0}, \\
\frac{d}{d t}\left(\mathbb{A}_{0} u+\sum_{j=1}^{N} \mathbb{A}_{j}(u)\right)+\mathbb{L} u & =\mathbb{F}(u), & u(0)=u_{0}, \\
\frac{d}{d t}\left(\mathbb{A}_{0} u+\sum_{j=1}^{N} \mathbb{A}_{j}(u)\right)+\mathbb{D P}(u) & =\mathbb{F}(u), & u(0)=u_{0}, \\
\frac{d}{d t}\left(\mathbb{A}_{0} u+\mathbb{L} u\right)+\mathbb{L} u+\mathbb{D P}(u) & =\mathbb{F}(u), & & u(0)=u_{0},
\end{array}
$$


где $\mathbb{A}_{0} u+\sum_{j=1}^{N} \mathbb{A}_{j}(u)$ - нелинейный эллиптический оператор, $\mathbb{L} u$ - линейный диссипативный эллиптический оператор, $\mathbb{D P}(u)$ - оператор “конвективной" нелинейности, $\mathbb{F}(u)$ - оператор нелинейного источника. В целях определенной завершенности цикла результатов о разрушении решений сильно нелинейных уравнений псевдопараболического типа возникла задача (1.1), имеющая физические приложения. В частности, к задаче (1.1) сводятся задачи о волновых процессах в полупроводниках или магнетиках при наличии внешнего электрического поля или магнитного поля при нелинейной зависимости диэлектрической проницаемости от электрического поля либо магнитной восприимчивости от магнитного поля, при учете диссипации в среде и наличии внешних источников. Подробный вьвод модельных уравнений приведен в [1], [2].

Принципиально новый подход, называемый методом пробных функиий и предложенный в работе [14], может быть применен для исследования дифференциальных неравенств псевдопараболического типа.

Отметим, что исследованию волновых квазилинейных диссипативных уравнений с источниками и без источников посвящены работы [15]-[21]. В этих работах рассматривались вопросы асимптотического поведения решений псевдопараболических уравнений при больших временах, а также вопросы сушествования решений типа уединенных волн и их устойчивости.

Приведем примеры некоторых модельных трехмерных сильно нелинейных уравнений типа Соболева, вывод которых содержится в работах [1], [2]:

$$
\begin{gathered}
\frac{\partial}{\partial t}\left(\Delta u-u+\sum_{j=1}^{N} \operatorname{div}\left(|\nabla u|^{p_{j}-2} \nabla u\right)\right)-u+u \frac{\partial u}{\partial x_{1}}+u^{3}=0 \\
\frac{\partial}{\partial t}\left(\Delta u-u-|u|^{q_{1}} u\right)+\Delta u+\frac{\partial|u|^{q_{2}+1}}{\partial x_{1}}+|u|^{2 q_{2}} u=0 \\
\frac{\partial}{\partial t}\left(-\Delta^{2} u+\Delta u+\operatorname{div}\left(|\nabla u|^{p_{1}-2} \nabla u\right)\right)+\Delta u+u \frac{\partial u}{\partial x_{1}}-\operatorname{div}\left(|\nabla u|^{2} \nabla u\right)=0 \\
\frac{\partial}{\partial t}\left(-\Delta^{2} u+\Delta u+\operatorname{div}\left(|\nabla u|^{p_{1}-2} \nabla u\right)\right)-\operatorname{div}\left(|\nabla u|^{2} \nabla u\right)+\Delta u \\
+\beta_{1} \frac{\partial}{\partial x_{1}}\left(\frac{\partial u}{\partial x_{2}} \frac{\partial u}{\partial x_{3}}\right)+\beta_{2} \frac{\partial}{\partial x_{2}}\left(\frac{\partial u}{\partial x_{3}} \frac{\partial u}{\partial x_{1}}\right)+\beta_{3} \frac{\partial}{\partial x_{3}}\left(\frac{\partial u}{\partial x_{1}} \frac{\partial u}{\partial x_{2}}\right)=0
\end{gathered}
$$

где $\beta_{1}+\beta_{2}+\beta_{3}=0,\left|\beta_{1}\right|+\left|\beta_{2}\right|+\left|\beta_{3}\right|>0, p_{j}>2, q_{1}, q_{2} \geqslant 0, p_{1}, p_{2}>2$.

\section{§ 2. Первоначальные определения и условия}

В настоящей статье мы будем в основном использовать стандартные определения функционального анализа, приведя лишь необходимые для дальнейшего изложения материала определения, предложения и вспомогательные результаты.

Пусть банаховы пространства $\mathbb{V}_{i}, \mathbb{V}_{0}, \mathbb{W}_{0}, \mathbb{W}_{1}$ бесконечномерны, сепарабельны и рефлексивны с соответствующими сопряженными банаховыми пространствами $\mathbb{V}_{i}^{*}, \mathbb{V}_{0}^{*}, \mathbb{W}_{0}^{*}, \mathbb{W}_{1}^{*}$ относительно соответствуюших скобок двойственности $\langle\cdot, \cdot\rangle_{i}$, 
$\langle\cdot, \cdot\rangle_{0},(\cdot, \cdot)_{0},(\cdot, \cdot)_{1}, i=\overline{1, N}$. Банаховы пространства $\mathbb{W}_{j}, j=\overline{2,4}$, рефлексивны и сепарабельны. Обозначим через $\|\cdot\|_{j}, j=\overline{0, N}$, нормы банаховых пространств $\mathbb{V}_{j}$, а через $\|\cdot\|_{j}^{*}$ - нормы банаховых пространств $\mathbb{V}_{j}^{*}$. Нормы банаховых пространств $\mathbb{W}_{i}, i=\overline{0,4}$, обозначим через $|\cdot|_{i}$, а нормы банаховых пространств $\mathbb{W}_{i}^{*}$ - через $|\cdot|_{i}^{*}$. Пусть $\mathbb{H}$ - гильбертово пространство, отождествленное с сопряженным.

Далее мы предположим, что выполнены следуюшие условия $\mathbb{V}$.

1) Банаховы пространства $\mathbb{V}_{i}, i=\overline{0, N}$, непрерывно вложены в $\mathbb{H}$.

2) Для сильно сопряженного к банахову относительно нормы $\|\cdot\|=\sum_{i=0}^{N}\|\cdot\|_{i}$ рефлексивного пространства $\mathbb{V} \equiv \bigcap_{i=0}^{N} \mathbb{V}_{i}$ справедливо явное выражение

$$
\mathbb{V}^{*}=\bigcup_{i=0}^{N} \mathbb{V}_{i}^{*} .
$$

3) Справедливы следуюшие цепочки непрерывных вложений:

$$
\begin{array}{ll}
\mathbb{V} \subseteq \mathbb{V}_{i} \subseteq \mathbb{H} \subseteq \mathbb{V}_{i}^{*} \subseteq \mathbb{V}^{*}, & i=\overline{0, N} \\
\mathbb{V} \subseteq \mathbb{W}_{j} \subseteq \mathbb{H} \subseteq \mathbb{W}_{j}^{*} \subseteq \mathbb{V}^{*}, & j=\overline{0,1} .
\end{array}
$$

4) Пространство $\mathbb{V}$ бесконечномерное и сепарабельное.

ЗАмечАниЕ 2.1. Из условия $\mathbb{V}, 1)$ вытекает, что пересечение $\mathbb{V} \equiv \bigcap_{i=0}^{N} \mathbb{V}_{i}$, наделенное нормой $\|\cdot\|=\sum_{i=0}^{N}\|\cdot\|_{i}$, является банаховым пространством.

Обозначим через $\langle\cdot, \cdot\rangle$ скобки двойственности между банаховыми пространствами $\mathbb{V}$ и $\mathbb{V}^{*}$.

ОПРЕДЕЛЕНИЕ 2.1. Оператор $\mathbb{A}: D \subset \mathbb{X} \rightarrow \mathbb{X}^{*}$ называется полунепрерывным на $D$, если для любых $u, v, w \in D$ функция $\lambda \rightarrow\langle\mathbb{A}(u+\lambda v), w\rangle$ непрерывна как функция из $\mathbb{R}^{1}$ в $\mathbb{R}^{1}$.

ОПРЕДЕЛЕНИЕ 2.2. Симметрическим назьвается линейньй оператор $\mathbb{A}$, действующий из рефлексивного банахова пространства $\mathbb{X}$ в сопряженное $\mathbb{X}^{*}$, если для любых $u, v \in \mathbb{X}$ выполнено соотношение

$$
\langle\mathbb{A} u, v\rangle=\langle\mathbb{A} v, u\rangle
$$

Пусть операторы $\mathbb{A}_{i}, \mathbb{A}_{0}, \mathbb{F}, \mathbb{L}$ определены на пространствах $\mathbb{V}_{i}, \mathbb{V}_{0}, \mathbb{W}_{0}, \mathbb{W}_{1}$ со значениями в соответствующих сопряженных пространствах:

$$
\mathbb{A}_{i}: \mathbb{V}_{i} \rightarrow \mathbb{V}_{i}^{*}, \quad i=\overline{1, N}, \quad \mathbb{A}_{0}: \mathbb{V}_{0} \rightarrow \mathbb{V}_{0}^{*}, \quad \mathbb{F}: \mathbb{W}_{0} \rightarrow \mathbb{W}_{0}^{*}, \quad \mathbb{L}: \mathbb{W}_{1} \rightarrow \mathbb{W}_{1}^{*},
$$

причем области значений операторов $\mathbb{A}_{j}, \mathbb{L}_{1}$ совпадают с соответствующими банаховыми пространствами $\mathbb{V}_{j}^{*}, j=\overline{0, N}, \mathbb{W}_{1}^{*}$.

Введем некоторые условия $\mathbb{A}$, налагаемые на операторные коэффициенты уравнения (1.1). 
1) Операторы $\mathbb{A}_{j}: \mathbb{V}_{j} \rightarrow \mathbb{V}_{j}^{*}, j=\overline{1, N}$, являются монотонными и полунепрерывными.

2) Операторы $\mathbb{A}_{j}$ имеют симметрическую производную Фреше для любого фиксированного $u \in \mathbb{V}_{j}$ :

$$
\mathbb{A}_{j, u}^{\prime}(u): \mathbb{V}_{j} \rightarrow \mathcal{L}\left(\mathbb{V}_{j}, \mathbb{V}_{j}^{*}\right), \quad j=\overline{1, N},
$$

неотрицательно определенную, и, кроме того, производная Фреше является полунепрерывной в каждом $\mathbb{V}_{j}$ в том смысле, что, каковы бы ни были $u, v, h_{1}, h_{2} \in$ $D \subset \mathbb{V}_{j}, j=\overline{1, N}$, и $\lambda \in \mathbb{R}^{1}$, функция $\left\langle\mathbb{A}_{j, u}^{\prime}(u+\lambda v) h_{1}, h_{2}\right\rangle_{j}$ непрерывна как функция из $\mathbb{R}^{1}$ в $\mathbb{R}^{1}$ относительно $\lambda$.

3) Справедливы неравенства снизу и сверху:

$$
\left\|\mathbb{A}_{j}(u)\right\|_{j}^{*} \leqslant M_{j}\|u\|_{j}^{p_{j}-1}, \quad\left\langle\mathbb{A}_{j}(u), u\right\rangle_{j} \geqslant m_{j}\|u\|_{j}^{p_{j}}, \quad p_{j}>2, \quad j=\overline{1, N},
$$

где $M_{j}>0$ и $m_{j}>0$.

$\left.3^{*}\right)$ Величина $\left\langle\mathbb{A}_{j}(u), u\right\rangle_{j}^{1 / p_{j}}$ является полунормой на $\mathbb{V}_{j}$.

4) Операторы $\mathbb{A}_{j}$ положительно однородные порядка $p_{j}-1$ :

$$
\mathbb{A}_{j}(s v)=s^{p_{j}-1} \mathbb{A}_{j}(v), \quad s>0 .
$$

Введем условия $\mathbb{A}_{0}$, налагаемые на операторные коэффициенты уравнения (1.1).

1) Справедливо неравенство $\left\langle\mathbb{A}_{0} u-\mathbb{A}_{0} v, u-v\right\rangle_{0} \geqslant m_{0}\|u-v\|_{0}^{2}$ для любых $u, v \in \mathbb{V}_{0}$, где $m_{0}>0$ - постоянная.

2) Оператор $\mathbb{A}_{0}$ симметричен.

3) Оператор $\mathbb{A}_{0}: \mathbb{V}_{0} \rightarrow \mathbb{V}_{0}^{*}$ удовлетворяет условию $\|\mathbb{A} u\|_{0}^{*} \leqslant M_{0}\|u\|_{0}$, где $M_{0}>0$ - постоянная.

$\left.3^{*}\right)$ Величина $\langle\mathbb{A} u, u\rangle_{0}$ является полунормой для $\mathbb{V}_{0}$.

Введем условия $\mathbb{F}$, налагаемые на операторные коэффициенты уравнения (1.1).

1) Оператор $\mathbb{F}(u): \mathbb{W}_{0} \rightarrow \mathbb{W}_{0}^{*}$ является ограниченно непрерывным по Липшицу:

$$
|\mathbb{F}(u)-\mathbb{F}(v)|_{0}^{*} \leqslant \mu_{1}(R)|u-v|_{0}, \quad R=\max \left(|u|_{0},|v|_{0}\right)
$$

где $\mu_{1}(R)$ - возрастающая и непрерывная функция.

2) Оператор $\mathbb{F}(u)$ дифференцируем по Фреше и имеет симметрическую производную при любом фиксированном $u \in \mathbb{W}_{0}$.

3) Для любых $s>0$ и $v \in \mathbb{W}_{0}$ выполнено равенство $\mathbb{F}(s v)=s^{1+q} \mathbb{F}(v), q>0$.

4) Справедливо неравенство сверху:

$$
|\mathbb{F}(u)|_{0}^{*} \leqslant B|u|_{0}^{q+1}, \quad B>0 .
$$

5) Справедливо неравенство снизу:

$$
(\mathbb{F}(u), u)_{0} \geqslant m_{\mathbb{F}}|u|_{0}^{q+2}
$$

Введем условия $\mathbb{L}$, налагаемые на операторные коэффициенты уравнения (1.1). 
1) Справедливо неравенство $(\mathbb{L} u-\mathbb{L} v, u-v)_{1} \geqslant d_{1}|u-v|_{1}^{2}$ для любых $u, v \in \mathbb{W}_{1}$, где $d_{1}>0$ - постоянная.

2) Оператор $\mathbb{L}$ симметричен.

3) Оператор $\mathbb{L}: \mathbb{W}_{1} \rightarrow \mathbb{W}_{1}^{*}$ удовлетворяет условию $|\mathbb{L} u|_{1}^{*} \leqslant D_{1}|u|_{1}$, где $D_{1}>0$ постоянная.

Введем условия $\mathbb{D P}$, налагаемые на операторные коэффициенты уравнения (1.1).

1) Оператор $\mathbb{D}: \mathbb{W}_{3} \rightarrow \mathbb{W}_{4} \subseteq \mathbb{V}_{0}^{*}$ является линейным, ограниченным и $\mathbb{D}(\mathbf{0})=\mathbf{0}$.

2) Оператор $\mathbb{P}(u): \mathbb{V}_{0} \subseteq \mathbb{W}_{2} \rightarrow \mathbb{W}_{3}$ является ограниченно непрерывным по Липшицу:

$$
\left|\mathbb{P}\left(u_{1}\right)-\mathbb{P}\left(u_{2}\right)\right|_{3} \leqslant \mu_{2}(R)\left|u_{1}-u_{2}\right|_{2},
$$

где $\mu_{2}(R)$ - непрерывная возрастающая функция.

3) Справедливо неравенство сверху: $|\mathbb{P}(u)|_{3} \leqslant B_{2}|u|_{2}^{(q+2) / 2}$.

4) Для любого $u \in \mathbb{V}_{0}$ имеет место равенство $\langle\mathbb{D P}(u), u\rangle_{0}=0$.

Отметим, что из условий $\mathbb{A}_{0}$ и $\mathbb{L}$ вытекает, что величины $\left\langle\mathbb{A}_{0} u, u\right\rangle_{0}^{1 / 2}$ и $(\mathbb{L} u, u)_{1}^{1 / 2}$ являются нормами на пространствах $\mathbb{V}_{0}$ и $\mathbb{W}_{1}$ соответственно.

Далее нам понадобится следующее вспомогательное утверждение.

УТВЕРЖДЕНИЕ 2.1. Для любъх $u, v \in \mathbb{V}_{0} \subset \mathbb{W}_{0} \subseteq \mathbb{W}_{2}$ справедливо неравенство

$$
\left|\langle\mathbb{D P}(u), v\rangle_{0}\right| \leqslant C\left\langle\mathbb{A}_{0} v, v\right\rangle_{0}^{1 / 2}(\mathbb{F}(u), u)_{0}^{1 / 2}, \quad C>0 .
$$

ДоказАТЕльство. Далее через $C$ мы будем обозначать различные постоянные. Справедлива следуюшая цепочка неравенств:

$$
\begin{aligned}
\left|\langle\mathbb{D P}(u), v\rangle_{0}\right| & \leqslant\|\mathbb{D}(\mathbb{P}(u))\|_{0}^{*}\|v\|_{0} \leqslant C\left\langle\mathbb{A}_{0} v, v\right\rangle_{0}^{1 / 2}|\mathbb{D}(\mathbb{P}(u))|_{4} \\
& \leqslant C\left\langle\mathbb{A}_{0} v, v\right\rangle_{0}^{1 / 2}|\mathbb{P}(u)|_{3} \leqslant C\left\langle\mathbb{A}_{0} v, v\right\rangle_{0}^{1 / 2}|u|_{2}^{(q+2) / 2} \\
& \leqslant C\left\langle\mathbb{A}_{0} v, v\right\rangle_{0}^{1 / 2}|u|_{0}^{(q+2) / 2} \leqslant C\left\langle\mathbb{A}_{0} v, v\right\rangle_{0}^{1 / 2}(\mathbb{F}(u), u)_{0}^{1 / 2} .
\end{aligned}
$$

Утверждение доказано.

Без ограничения общности будем считать, что в (2.2) постоянная $C$ равна единице.

\section{§3. Слабая обобщенная разрешимость, единственность и разрушение решения задачи (1.1)}

ОПРЕДЕЛЕНИЕ 3.1. Слабым обобщенным решением абстрактной задачи Коши (1.1) назьвается решение задачи, удовлетворяюшее условиям

$$
\begin{gathered}
\frac{d}{d t}\left\langle\mathbb{A}_{0} u, v\right\rangle_{0}+\frac{d}{d t} \sum_{j=1}^{N}\left\langle\mathbb{A}_{j}(u), v\right\rangle_{j}+\langle\mathbb{D P}(u), v\rangle_{0}+(\mathbb{L} u, v)_{1}=(\mathbb{F}(u), v)_{0} \\
\forall v \in \mathbb{V}, \quad u(0)=u_{0} \in \mathbb{V}
\end{gathered}
$$

где производная по времени понимается в смысле $\mathcal{D}^{\prime}(0, T)$. Поскольку решение $u(t)$ ищется в классе $\mathbb{L}^{\infty}(0, T ; \mathbb{V}), \quad \frac{d u}{d t} \in \mathbb{L}^{2}\left(0, T ; \mathbb{V}_{0}\right)$, то имеют место включения $\mathbb{A}_{0} u \in \mathbb{L}^{\infty}\left(0, T ; \mathbb{V}_{0}^{*}\right), \mathbb{A}_{j}(u) \in \mathbb{L}^{\infty}\left(0, T ; \mathbb{V}_{j}^{*}\right), \mathbb{D P}(u) \in \mathbb{L}^{\infty}\left(0, T ; \mathbb{W}_{4}\right)$, 
$\mathbb{L} u \in \mathbb{L}^{\infty}\left(0, T ; \mathbb{W}_{1}^{*}\right), \mathbb{F}(u) \in \mathbb{L}^{\infty}\left(0, T ; \mathbb{W}_{0}^{*}\right)$, Тем самым задача $(3.1)$ в силу условий $\mathbb{V}$ и условий следуюшей ниже теоремы 3.1 эквивалентна задаче

$$
\begin{gathered}
\frac{d}{d t}\left\langle\mathbb{A}_{0} u, v\right\rangle+\frac{d}{d t} \sum_{j=1}^{N}\left\langle\mathbb{A}_{j}(u), v\right\rangle+\langle\mathbb{D P}(u), v\rangle+\langle\mathbb{L} u, v\rangle=\langle\mathbb{F}(u), v\rangle \\
\forall v \in \mathbb{V}, \quad u(0)=u_{0} \in \mathbb{V},
\end{gathered}
$$

где производная по времени понимается в смысле $\mathcal{D}^{\prime}(0, T)$.

Справедлива следующая теорема.

ТЕОРема 3.1. Пусть выполнены условия $\mathbb{A}, \mathbb{A}_{0}, \mathbb{F}, \mathbb{L}, \mathbb{D P}$. Предположим, что $\mathbb{V} \hookrightarrow \mathbb{W}_{0} u \mathbb{V} \hookrightarrow \mathbb{W}_{1}$, т.е. вложения вполне непрерывны, и что $\mathbb{V}_{0} \subset \mathbb{W}_{1}$ $u \mathbb{V}_{0} \subset \mathbb{W}_{0} \subseteq \mathbb{W}_{2}$. Кроме того, предположим, что найдется $j^{*} \in \overline{1, N}$, для которого $p_{j^{*}} \geqslant p_{j}$ u $p_{j}^{*} \geqslant q+2, j=\overline{1, N}$, такое, что $\mathbb{V}_{j^{*}} \subseteq \mathbb{W}_{0}$. Тогда для любого $u_{0} \in \mathbb{V}$ найдется максимальное $T_{0} \equiv T_{u_{0}}>0$ такое, что для любого $0<T<T_{0}$ задача Коши (1.1) имеет единственное непродолжаемое во времени решение $u(t)$ класса $\mathbb{L}^{\infty}(0, T ; \mathbb{V}), \quad \frac{d}{d t} u(t) \in \mathbb{L}^{2}\left(0, T ; \mathbb{V}_{0}\right)$, m. е. либо $T_{0}=+\infty$, либо $T_{0}<+\infty$, в в последнем случае имеет место предельное равенство

$$
\lim _{t \uparrow T_{0}}\|u\|_{\mathbb{V}}=+\infty
$$

причем для положстельно определенной в силу условий $\left.\left.\mathbb{A}_{0}, 2\right) u \mathbb{A}, 3\right) u$ имеющей смысл кинетической әнергии функиии

$$
\Phi(t) \equiv \frac{1}{2}\left\langle\mathbb{A}_{0} u, u\right\rangle_{0}+\sum_{j=1}^{N} \frac{p_{j}-1}{p_{j}}\left\langle\mathbb{A}_{j}(u), u\right\rangle_{j}
$$

в трех случаях относительно возможных значений величины $\alpha \equiv \frac{q+2}{\bar{p}}, \bar{p} \equiv$ $\max _{j=\overline{1, N}} p_{j}$, справедливь следующие оченки:

1) если $\alpha=1$, то $T_{0}=+\infty u \Phi(t) \leqslant \Phi_{0} \exp \left\{C_{2} t\right\}$;

2) если $\alpha \in(0,1)$, то $T_{0}=+\infty u \Phi(t) \leqslant \Phi_{0}\left[1+(1-\alpha) C_{2} \Phi_{0}^{\alpha-1} t\right]^{1 /(1-\alpha)}$;

3) если $\alpha>1$ и выполнены неравенства

$$
\Phi^{\prime}(0)>\frac{\beta}{\alpha-1} \Phi(0), \quad \Phi^{\prime}(0)>\sqrt{\frac{\gamma}{\alpha-1}} \Phi(0),
$$

то найдется такое $T_{0}>0$, что

$$
\lim _{t \uparrow T_{0}} \Phi(t)=+\infty, \quad T_{1} \leqslant T_{0} \leqslant T_{2},
$$


əде

$$
\begin{gathered}
T_{1}=\frac{2}{q} \Phi_{0}^{-q / 2} \bar{B}^{-1}, \quad T_{2}=\Phi_{0}^{1-\alpha} A^{-1}, \\
C_{2} \equiv B C_{j^{*}}^{q+2}\left(\frac{\bar{p}}{\bar{p}-1}\right)^{\alpha}, \quad|v|_{0} \leqslant C_{j^{*}}\left\langle\mathbb{A}_{j^{*}} v, v\right\rangle_{j^{*}}^{1 / p_{j^{*}}}, \\
\Phi_{0} \equiv \Phi(0), \quad \Phi^{\prime}(0) \equiv\left(\mathbb{F}\left(u_{0}\right), u_{0}\right)_{0}-\left(\mathbb{L} u_{0}, u_{0}\right)_{1}, \\
\alpha \equiv \frac{\bar{p}+q+2}{2 \bar{p}}, \quad \beta \equiv \frac{(q+2)(q+1)}{q+2-\bar{p}}, \\
\gamma \equiv \frac{4(q+1)^{2}}{q+2-\bar{p}}, \quad q+2>\bar{p} \equiv \max _{j=\overline{1, n}} p_{j}, \\
A^{2} \equiv(\alpha-1)^{2} \Phi_{0}^{-2 \alpha}\left[\left(\Phi^{\prime}(0)\right)^{2}-\frac{\gamma}{\alpha-1} \Phi^{2}(0)\right] \\
\bar{B}=2^{q+2} B C_{1}^{q+2},
\end{gathered}
$$

$C_{1}$ - константа наилучшего вложения $\mathbb{V} \hookrightarrow \mathbb{W}_{0}, \quad B$ - константа из условия $\mathbb{F}, 4)$.

ДокАЗАТЕЛЬСТвО. Проведем доказательство в несколько этапов.

ЗАмЕчАниЕ 3.1 . В силу принадлежности решения $u(t)$ классу $\mathbb{L}^{\infty}(0, T ; \mathbb{V})$, $\frac{d u}{d t} \in \mathbb{L}^{2}\left(0, T ; \mathbb{V}_{0}\right)$, следует, что после возможного изменения на множестве нулевой меры Лебега отображение $u(t):[0, T] \rightarrow \mathbb{V}_{0}$ непрерывно. Значит, имеет смысл начальное условие $u(0)=u_{0}$.

Шаг 1. Приближения Галёркина. Из сепарабельности пространства $\mathbb{V}$ следует, что найдется счетная всюду плотная в $\mathbb{V}$ линейно независимая система функций $\left\{w_{i}\right\}_{i=1}^{m} \hookrightarrow \mathbb{V}$. Докажем разрешимость задачи (3.1) методом Галёркина в сочетании с методами монотонности и компактности [26].

Сначала рассмотрим следуюшую конечномерную аппроксимацию задачи (3.1):

$$
\begin{gathered}
\frac{d}{d t}\left\langle\mathbb{A}_{0} u_{m}, w_{k}\right\rangle_{0}+\frac{d}{d t} \sum_{j=1}^{N}\left\langle\mathbb{A}_{j}\left(u_{m}\right), w_{k}\right\rangle_{j}+\left(\mathbb{L} u_{m}, w_{k}\right)_{1} \\
+\left\langle\mathbb{D P}\left(u_{m}\right), w_{k}\right\rangle_{0}=\left(\mathbb{F}\left(u_{m}\right), w_{k}\right)_{0}, \quad k=\overline{1, m}, \\
u_{m}=\sum_{i=1}^{m} c_{m i}(t) w_{i}, \quad u_{m 0}=\sum_{i=1}^{m} c_{m i}(0) w_{i}, \\
c_{m i}(0)=\alpha_{m i}, \quad u_{m 0} \rightarrow u_{0} \quad \text { сильно в } \mathbb{V} .
\end{gathered}
$$

Из $(3.2)$ вытекает, что в классе $\mathbb{C}^{1}\left(\left[0, T_{m}\right] ; \mathbb{V}\right)$ для функции $u_{m}$ справедлива система уравнений

$$
\begin{aligned}
& \sum_{i=1}^{m} c_{m i}^{\prime} {\left[\left\langle\mathbb{A}_{0} w_{i}, w_{k}\right\rangle_{0}+\sum_{j=1}^{N}\left\langle\mathbb{A}_{j, u_{m}}^{\prime}\left(u_{m}\right) w_{i}, w_{k}\right\rangle_{j}\right] } \\
&+\left\langle\mathbb{D P}\left(u_{m}\right), w_{k}\right\rangle_{0}+\left(\mathbb{L} u_{m}, w_{k}\right)_{1}=\left(\mathbb{F}\left(u_{m}\right), w_{k}\right)_{0}, \quad k=\overline{1, m} .
\end{aligned}
$$


Введем обозначение

$$
a_{i k} \equiv\left\langle\mathbb{A}_{0} w_{i}, w_{k}\right\rangle_{0}+\sum_{j=1}^{N}\left\langle\mathbb{A}_{j, u_{m}}^{\prime}\left(u_{m}\right) w_{i}, w_{k}\right\rangle_{j}
$$

Очевидно,

$$
\sum_{i, k=1}^{m} a_{i k} \xi_{i} \xi_{k}=\left\langle\mathbb{A}_{0} \eta, \eta\right\rangle_{0}+\sum_{j=1}^{N}\left\langle\mathbb{A}_{j, u_{m}}^{\prime}\left(u_{m}\right) \eta, \eta\right\rangle_{j} \geqslant\left\langle\mathbb{A}_{0} \eta, \eta\right\rangle_{0}, \quad \eta=\sum_{i=1}^{m} \xi_{i} w_{i}
$$

поскольку $\mathbb{A}_{0}$ - положительно определенньй оператор, а значит, $\left\langle\mathbb{A}_{0} \eta, \eta\right\rangle_{0} \geqslant 0$, причем равенство $\left\langle\mathbb{A}_{0} \eta, \eta\right\rangle_{0}=0$ имеет место тогда и только тогда, когда $\eta=0$. С другой стороны,

$$
\eta \equiv \sum_{i=1}^{m} \xi_{i} w_{i}
$$

и в силу линейной независимости системы функций $\left\{w_{i}\right\}_{i=1}^{m}$ в $\mathbb{V}$ приходим к выводу, что $\eta=0$ тогда и только тогда, когда $\left\{\xi_{i}\right\}_{i=1}^{m}=\mathbf{0}$. Отсюда и из работы [27] получим, что $\operatorname{det}\left\{a_{i k}\right\}_{i, k=1}^{m}>0$.

Докажем теперь, что функционалы $f_{j} \equiv\left\langle\mathbb{A}_{j, u_{m}}^{\prime}\left(u_{m}\right) w_{i}, w_{k}\right\rangle_{j}$ являются непрерьвными по совокупности переменных $c_{m i}, i=\overline{1, m}$. Действительно, пусть $\left(\bar{c}_{m 1}, \ldots, \bar{c}_{m m}\right)$ - некоторая точка евклидова пространства $\mathbb{R}^{m}$. Фиксируем произвольное $\varepsilon>0$. Справедливы следующие неравенства:

$$
\begin{aligned}
&\left|f_{j}\left(\bar{c}_{m 1}, \ldots, \bar{c}_{m m}\right)-f_{j}\left(c_{m 1}, \ldots, c_{m m}\right)\right| \\
& \leqslant\left|f_{j}\left(\bar{c}_{m 1}, \ldots, \bar{c}_{m m}\right)-f_{j}\left(c_{m 1}, \bar{c}_{m 2}, \ldots, \bar{c}_{m m}\right)\right| \\
&+\left|f_{j}\left(c_{m 1}, \bar{c}_{m 2}, \ldots, \bar{c}_{m m}\right)-f_{j}\left(c_{m 1}, c_{m 2}, \bar{c}_{m 3}, \ldots, \bar{c}_{m m}\right)\right| \\
&+\left|f_{j}\left(c_{m 1}, c_{m 2}, \bar{c}_{m 3}, \ldots, \bar{c}_{m m}\right)-f_{j}\left(c_{m 1}, c_{m 2}, c_{m 3}, \ldots, \bar{c}_{m m}\right)\right|+\ldots \\
& \quad \ldots+\left|f_{j}\left(c_{m 1}, c_{m 2}, \ldots, c_{(m-1) m}, \bar{c}_{m m}\right)-f_{j}\left(c_{m 1}, \ldots, c_{m m}\right)\right| .
\end{aligned}
$$

В силу условия $\mathbb{A}, 2)$ найдется такое $\delta(\varepsilon)>0$, что каждое слагаемое в правой части неравенства (3.4) при выполнении условия

$$
\sum_{k=1}^{m}\left|\bar{c}_{m k}-c_{m k}\right| \leqslant \delta(\varepsilon)
$$

меньше величины $\varepsilon /(m+1)$. Докажем теперь непрерывность по Липшищу функционалов $f_{0 k}=\left(\mathbb{F}\left(u_{m}\right), w_{k}\right)_{0}$ по совокупности переменных $\left\{c_{m i}\right\}_{i=1}^{m}$. Пусть $u_{j}=$ $\sum_{i=1}^{m} c_{m i}^{j} w_{j}, j=1,2$. В силу условия $\left.\mathbb{F}, 1\right)$ справедлива цепочка неравенств

$$
\left|\left(\mathbb{F}\left(u_{1}\right)-\mathbb{F}\left(u_{2}\right), w_{k}\right)_{0}\right| \leqslant\left|\mathbb{F}\left(u_{1}\right)-\mathbb{F}\left(u_{2}\right)\right|_{0}^{*}|w|_{0} \leqslant B\left|u_{1}-u_{2}\right|_{0} \leqslant B \sum_{l=1}^{m}\left|c_{m l}^{1}-c_{m l}^{2}\right|
$$


Тем самым функция $f_{0}=f_{0}\left(c_{m 1}, \ldots, c_{m m}\right)$ является непрерывной по Липшицу и, тем более, непрерывной по совокупности переменных.

Рассмотрим теперь функционалы $g_{k} \equiv\left\langle\mathbb{D P}\left(u_{m}\right), w_{k}\right\rangle_{0}$ от переменных $c_{m 1}$, $c_{m 2}, \ldots, c_{m m}$. Точно так же, как и при выводе предыдушего неравенства, в силу условий $\mathbb{D P}$ докажем непрерывность по Липшицу функционалов $g_{k}$. Стало быть, система обыкновенных дифференциальных уравнений (3.3) является системой типа Коши-Ковалевской и удовлетворяет условиям, гарантируюшим ее разрешимость на некотором сегменте $\left[0, T_{m}\right], T_{m}>0$, в классе $c_{m k}(t) \in \mathbb{C}^{1}\left(\left[0, T_{m}\right]\right)$, $k=\overline{1, m}$ (см., например, [28]).

Шаг 2. Априорные оценки. Докажем следующую лемму.

Лемма 3.1. Найдется $T>0$, не зависящее от $m \in \mathbb{N}$, такое, что для последовательности $\left\{u_{m}\right\}$ приближений Галёркина справедливь равномерно по $m \in \mathbb{N}$ следующие свойства:

1) $u_{m}$ ограничено в $\mathbb{L}^{\infty}(0, T ; \mathbb{V})$;

2) $u_{m}^{\prime}$ ограничено в $\mathbb{L}^{2}\left(0, T ; \mathbb{V}_{0}\right)$;

3) $\mathbb{A}_{0} u_{m}$ ограничено в $\mathbb{L}^{\infty}\left(0, T ; \mathbb{V}_{0}^{*}\right)$;

4) $\mathbb{A}_{j}\left(u_{m}\right)$ ограничено в $\mathbb{L}^{\infty}\left(0, T ; \mathbb{V}_{j}^{*}\right)$;

5) $\mathbb{F}\left(u_{m}\right)$ ограничено в $\mathbb{L}^{\infty}\left(0, T ; \mathbb{W}_{0}^{*}\right)$;

6) $\mathbb{L} u_{m}$ ограничено в $\mathbb{L}^{\infty}\left(0, T ; \mathbb{W}_{1}^{*}\right)$.

ДоКАЗАТЕЛЬСтво. Умножим обе части равенства $(3.3)$ на $c_{m k}(t)$ и просуммируем по $k=\overline{1, m}$; тогда получим

$$
\left\langle\mathbb{A}_{0} u_{m}^{\prime}, u_{m}\right\rangle_{0}+\sum_{j=1}^{N}\left\langle\mathbb{A}_{j, u_{m}}^{\prime}\left(u_{m}\right) u_{m}^{\prime}, u_{m}\right\rangle_{j}+\left(\mathbb{L} u_{m}, u_{m}\right)_{1}=\left(\mathbb{F}\left(u_{m}\right), u_{m}\right)_{0} .
$$

С другой стороны, в силу того, что $u_{m}=\sum_{k=1}^{N} c_{m k}(t) w_{k} \in \mathbb{C}^{1}\left(\left[0, T_{m}\right] ; \mathbb{V}\right)$, и условий $\mathbb{A}, 2), 4)$ справедливы равенства

$$
\begin{gathered}
\frac{d}{d t}\left\langle\mathbb{A}_{j}\left(u_{m}\right), u_{m}\right\rangle_{j}=p_{j}\left\langle\mathbb{A}_{j}\left(u_{m}\right), u_{m t}^{\prime}\right\rangle_{j}, \\
\left\langle\left(\mathbb{A}_{j}\left(u_{m}\right)\right)_{t}^{\prime}, u_{m}\right\rangle_{j}+\left\langle\mathbb{A}_{j}\left(u_{m}\right), u_{m t}^{\prime}\right\rangle_{j}=p_{j}\left\langle\mathbb{A}_{j}\left(u_{m}\right), u_{m t}^{\prime}\right\rangle_{j}, \\
\left\langle\left(\mathbb{A}_{j}\left(u_{m}\right)\right)_{t}^{\prime}, u_{m}\right\rangle_{j}=\left(p_{j}-1\right)\left\langle\mathbb{A}_{j}\left(u_{m}\right), u_{m t}^{\prime}\right\rangle_{j}=\frac{p_{j}-1}{p_{j}} \frac{d}{d t}\left\langle\mathbb{A}_{j}\left(u_{m}\right), u_{m}\right\rangle_{j} .
\end{gathered}
$$

Отсюда и из (3.5) получим

$$
\frac{d}{d t}\left[\frac{1}{2}\left\langle\mathbb{A}_{0} u_{m}, u_{m}\right\rangle_{0}+\sum_{j=1}^{N} \frac{p_{j}-1}{p_{j}}\left\langle\mathbb{A}_{j}\left(u_{m}\right), u_{m}\right\rangle_{j}\right]+\left(\mathbb{L} u_{m}, u_{m}\right)_{1}=\left(\mathbb{F}\left(u_{m}\right), u_{m}\right)_{0} .
$$

Интегрируя обе части соотношения (3.6) по $t \in\left(0, T_{m}\right)$, получим

$$
\begin{aligned}
\frac{1}{2}\left\langle\mathbb{A}_{0} u_{m}, u_{m}\right\rangle_{0}+\sum_{j=1}^{N} \frac{p_{j}-1}{p_{j}}\left\langle\mathbb{A}_{j}\left(u_{m}\right), u_{m}\right\rangle_{j}=\frac{1}{2}\left\langle\mathbb{A}_{0} u_{m 0}, u_{m 0}\right\rangle_{0} \\
+\sum_{j=1}^{N} \frac{p_{j}-1}{p_{j}}\left\langle\mathbb{A}_{j}\left(u_{m 0}\right), u_{m 0}\right\rangle_{j}+\int_{0}^{t}\left[\left(\mathbb{F}\left(u_{m}\right), u_{m}\right)_{0}-\left(\mathbb{L} u_{m}, u_{m}\right)_{1}\right] d s .
\end{aligned}
$$


В силу условия $\mathbb{A}, 3)$ в банаховом пространстве $\mathbb{V}$ можно выбрать норму, эквивалентную исходной:

$$
\|v\|=\frac{1}{2}\left\langle\mathbb{A}_{0} v, v\right\rangle_{0}^{1 / 2}+\sum_{j=1}^{N} \frac{p_{j}-1}{p_{j}}\left\langle\mathbb{A}_{j}(v), v\right\rangle_{j}^{1 / p_{j}}
$$

$\mathrm{C}$ другой стороны, $\mathbb{V} \hookrightarrow \mathbb{W}_{0}$ и $\left|\left(\mathbb{F}\left(u_{m}\right), u_{m}\right)_{0}\right| \leqslant B\left|u_{m}\right|_{0}^{q+2}$. Из соотношения (3.7) следует, что

$$
\begin{aligned}
\frac{1}{2}\left\langle\mathbb{A}_{0} u_{m}, u_{m}\right\rangle_{0}+\sum_{j=1}^{N} \frac{p_{j}-1}{p_{j}}\left\langle\mathbb{A}_{j}\left(u_{m}\right), u_{m}\right\rangle_{j} \\
\leqslant \frac{1}{2}\left\langle\mathbb{A}_{0} u_{m 0}, u_{m 0}\right\rangle_{0}+\sum_{j=1}^{N} \frac{p_{j}-1}{p_{j}}\left\langle\mathbb{A}_{j}\left(u_{m 0}\right), u_{m 0}\right\rangle_{j} \\
+2^{q+2} B C_{1}{ }^{q+2} \int_{0}^{t}\left(\frac{1}{2}\left\langle\mathbb{A}_{0} u_{m}, u_{m}\right\rangle_{0}+\sum_{j=1}^{N} \frac{p_{j}-1}{p_{j}}\left\langle\mathbb{A}_{j}\left(u_{m}\right), u_{m}\right\rangle_{j}\right)^{q+2} d s .
\end{aligned}
$$

Выведем теперь вторую априорную оценку. Умножим (3.3) на $c_{m k}^{\prime}$ и просуммируем по $k=\overline{1, m}$; в результате интегрирования обеих частей полученного равенства по $t \in\left(0, T_{m}\right)$ получим

$$
\begin{aligned}
& \int_{0}^{t}\left(\left\langle\mathbb{A}_{0} u_{m}^{\prime}, u_{m}^{\prime}\right\rangle_{0}+\sum_{j=1}^{N}\left\langle\left(\mathbb{A}_{j}\left(u_{m}\right)\right)^{\prime}, u_{m}^{\prime}\right\rangle_{j}\right) d s \\
& \quad+\frac{1}{q+2}\left(\mathbb{F}\left(u_{m 0}\right), u_{m 0}\right)_{0}-\frac{1}{2}\left(\mathbb{L} u_{m 0}, u_{m 0}\right)_{1} \\
&=\frac{1}{q+2}\left(\mathbb{F}\left(u_{m}\right), u_{m}\right)_{0}-\frac{1}{2}\left(\mathbb{L} u_{m}, u_{m}\right)_{1} \\
& \leqslant \frac{1}{2}\left(\mathbb{F}\left(u_{m}\right), u_{m}\right)_{0}-\frac{1}{2}\left(\mathbb{L} u_{m}, u_{m}\right)_{1}-\int_{0}^{t}\left\langle\mathbb{D P}\left(u_{m}\right), u_{m}^{\prime}\right\rangle_{0} d s .
\end{aligned}
$$

Введем обозначение

$$
\Phi_{m} \equiv \frac{1}{2}\left\langle\mathbb{A}_{0} u_{m}, u_{m}\right\rangle_{0}+\sum_{j=1}^{N} \frac{p_{j}-1}{p_{j}}\left\langle\mathbb{A}_{j}\left(u_{m}\right), u_{m}\right\rangle_{j}, \quad \Phi_{m 0} \equiv \Phi_{m}(0) .
$$

Из неравенства (3.9) получим

$$
\Phi_{m} \leqslant \Phi_{m 0}+\bar{B} \int_{0}^{t} \Phi_{m}^{1+q / 2}(s) d s
$$

где

$$
\bar{B} \equiv 2^{q+2} B C_{1}^{q+2}
$$


$C_{1}$ - константа наилучшего вложения $\mathbb{V} \hookrightarrow \mathbb{W}_{0}, B$ - константа из условия $\left.\mathbb{F}, 4\right)$. Теперь из неравенства (3.12) и теоремы Бихари (см., например, [29]) следует

$$
\Phi_{m} \leqslant \frac{\Phi_{m 0}}{\left[1-\frac{q}{2} \Phi_{m 0}^{q / 2} \bar{B} t\right]^{2 / q}} .
$$

Поскольку $u_{m 0} \rightarrow u_{0}$ сильно в $\mathbb{V}$, то $\Phi_{m 0} \leqslant C_{0}$, где $C_{0}$ не зависит от $m \in \mathbb{N}$. Далее возможны два случая для некоторой подпоследовательности последовательности $\left\{u_{m}\right\}:$ либо $\Phi_{m 0} \downarrow \Phi_{0}$, либо $\Phi_{m 0} \uparrow \Phi_{0}$.

Рассмотрим сначала случай $\Phi_{m 0} \uparrow \Phi_{0}$. Тогда справедливы неравенства

$$
\begin{gathered}
1-\frac{q}{2} \bar{B} \Phi_{m 0}^{q / 2} t \geqslant 1-\frac{q}{2} \bar{B} \Phi_{0}^{q / 2} t \quad \forall t \in(0, T), \quad T \in\left(0, T_{1}\right), \quad T_{1}=\frac{2}{q} \bar{B}^{-1} \Phi_{0}^{-q / 2}, \\
\Phi_{m} \leqslant C_{1} \quad \forall t \in(0, T), \quad T \in\left(0, T_{1}\right), \quad T_{1}=\frac{2}{q} \bar{B}^{-1} \Phi_{0}^{-q / 2},
\end{gathered}
$$

где $C_{1}$ не зависит от $m \in \mathbb{N}$.

Рассмотрим теперь случай $\Phi_{m 0} \downarrow \Phi_{0}$. Тогда для любого $\bar{m}<m$ имеем

$$
\Phi_{\bar{m} 0}>\Phi_{m 0}, \quad 1-\frac{q}{2} \bar{B} \Phi_{m 0}^{q / 2} t \geqslant 1-\frac{q}{2} \bar{B} \Phi_{\bar{m} 0}^{q / 2} t
$$

и для любого $t \in\left[0, \bar{B}^{-1} \Phi_{\bar{m} 0}^{-(q+1)}\right)$ получим

$$
\Phi_{m} \leqslant \frac{C_{0}}{\left[1-\frac{q}{2} \Phi_{\bar{m} 0}^{q / 2} \bar{B} t\right]^{2 / q}} .
$$

Значит, для любого фиксированного $T \in\left(0, T_{1}\right)$ найдется такое $\bar{m} \in \mathbb{N}$, что будет иметь место неравенство (3.14).

В силу того, что всегда найдется $p_{j^{*}}=\max _{j=\overline{1, N}} p_{j}>2, p_{j^{*}} \geqslant q+2$, такое, что $\mathbb{V}_{j^{*}} \subset \mathbb{W}_{0}$, справедливо следующее неравенство:

$$
|v|_{0} \leqslant C_{j^{*}}\left\langle\mathbb{A}_{j^{*}}(v), v\right\rangle_{j^{*}}^{1 / p_{j^{*}}}
$$

Из соотношения (3.15) сразу получаем, что

$$
|v|_{0}^{q+2} \leqslant C_{j^{*}}^{q+2}\left(\frac{p_{j^{*}}}{p_{j^{*}}-1}\right)^{(q+2) / p_{j^{*}}}\left(\frac{1}{2}\left\langle\mathbb{A}_{0} v, v\right\rangle_{0}+\sum_{j=1}^{N} \frac{p_{j}-1}{p_{j}}\left\langle\mathbb{A}_{j}(v), v\right\rangle_{j}\right)^{(q+2) / p_{j^{*}}} .
$$

С учетом соотношений (3.7), (3.11), (3.15) и (3.16) приходим к выводу:

$$
\Phi_{m} \leqslant \Phi_{m 0}+B C_{j^{*}}^{q+2}\left(\frac{p_{j^{*}}}{p_{j^{*}}-1}\right)^{(q+2) / p_{j^{*}}} \int_{0}^{t} \Phi_{m}^{\alpha_{j^{*}}}(s) d s, \quad \alpha_{j^{*}} \equiv \frac{q+2}{p_{j^{*}}} .
$$


О РАЗРУШЕНИИ РЕШЕНИЙ КЛАССА НЕЛИНЕЙНЫХ ВОЛНОВЫХ УРАВНЕНИЙ 101

Рассмотрим два случая: $\alpha_{j^{*}}<1$ и $\alpha_{j^{*}}=1$. Воспользовавшись теоремами Гронуолла-Белмана-Бихари [29], получим

$$
\begin{aligned}
& \Phi_{m} \leqslant\left[\Phi_{m 0}^{1-\alpha_{j^{*}}}+\left(1-\alpha_{j^{*}}\right) C_{2} t\right]^{1 /\left(1-\alpha_{j^{*}}\right)}, \quad \alpha_{j^{*}} \in(0,1) \\
& \Phi_{m} \leqslant \Phi_{m 0} \exp \left\{C_{2} t\right\}, \quad \alpha_{j^{*}}=1
\end{aligned}
$$

где

$$
C_{2} \equiv B C_{j^{*}}^{q+2}\left(\frac{p_{j^{*}}}{p_{j^{*}}-1}\right)^{(q+2) / p_{j^{*}}} .
$$

Вследствие сильной сходимости $u_{m 0} \rightarrow u_{0}$ в $\mathbb{V}$ имеем $\Phi_{m 0} \leqslant C_{0}$, где $C_{0}$ не зависит от $m \in \mathbb{N}$. Поэтому получим

$$
\Phi_{m 0} \leqslant C_{3} \quad \forall T \geqslant 0, \quad \forall \alpha_{j^{*}} \in(0,1]
$$

Из условия $\mathbb{A}, 3)$ и соотношений $(3.12),(3.14),(3.20)$ следует, что

$$
\begin{aligned}
\left(\mathbb{F}\left(u_{m}\right), u_{m}\right)_{0} & \leqslant B\left|u_{m}\right|_{0}^{q+2} \\
& \leqslant 2^{q+2} B C_{1}^{q+2}\left(\frac{1}{2}\left\langle\mathbb{A}_{0} u_{m}, u_{m}\right\rangle_{0}+\sum_{j=1}^{N} \frac{p_{j}-1}{p_{j}}\left\langle\mathbb{A}_{j}\left(u_{m}\right), u_{m}\right\rangle_{j}\right)^{q+2} \\
& \leqslant C_{3} \Phi_{m}^{(q+2) / 2} \leqslant C_{30},
\end{aligned}
$$

где $0<C_{30}<+\infty$ - не зависяшая от $m \in \mathbb{N}$ постоянная для любых $T>0$ в случае $\alpha_{j^{*}} \in(0,1]$ и для любых $T \in\left(0, T_{1}\right), T_{1}$ определено в $(3.16)$, в случае $\alpha_{j^{*}}>1$. Теперь из (3.10) и условий $\mathbb{A}, 2)$ и $\left.\mathbb{A}_{0}, 2\right)$ вытекает, что

$$
m_{0} \int_{0}^{t}\left\|u_{m}^{\prime}\right\|_{0}^{2} d s \leqslant \int_{0}^{t}\left\langle\mathbb{A}_{0} u_{m}^{\prime}, u_{m}^{\prime}\right\rangle_{0} d s \leqslant C_{40},
$$

где $0<C_{40}<+\infty$ - не зависяшая от $m \in \mathbb{N}$ постоянная для любых $T>0$ в случае $\alpha_{j^{*}} \in(0,1]$ и для любых $T \in\left(0, T_{1}\right), T_{1}$ определено в $(3.14)$, в случае $\alpha_{j^{*}}>1$.

Итак, выполнены свойства 1)-6) леммы 3.1. Эти свойства имеют место для любых $T>0$ в случае $\alpha_{j^{*}} \in(0,1]$ и для любых $T \in\left(0, T_{1}\right), T_{1}$ определено в $(3.14)$, в случае $\alpha_{j *}>1$. Лемма доказана.

Найдется такая подпоследовательность последовательности $\left\{u_{m}\right\}$ (обозначим ее так же), что

$$
u_{m} \rightarrow u \quad \text { *-слабо в } \mathbb{L}^{\infty}(0, T ; \mathbb{V}), \quad u_{m}^{\prime} \rightarrow u^{\prime} \quad \text { слабо в } \mathbb{L}^{2}\left(0, T ; \mathbb{V}_{0}\right)
$$

Из (3.22) и компактного вложения $\mathbb{V} \hookrightarrow \mathbb{W}_{0}$ получим, что для почти всех $t \in$ $(0, T)$ имеет место сильная сходимость $u_{m}(t) \rightarrow u(t)$ в $\mathbb{W}_{0}$. С другой стороны, в силу условия $\mathbb{F}, 1)$ имеем

$$
\left|\mathbb{F}\left(u_{m}\right)-\mathbb{F}(u)\right|_{0}^{*} \leqslant \mu(R)\left|u_{m}-u\right|_{0} \rightarrow 0, \quad m \rightarrow+\infty, \quad R=\max \left\{|u|_{0},\left|u_{m}\right|_{0}\right\} .
$$


Отсюда вытекает сильная сходимость

$$
\mathbb{F}\left(u_{m}\right)(t) \rightarrow \mathbb{F}(u)(t) \quad \text { в } \mathbb{W}_{0}^{*} \text { для почти всех } t \in(0, T) .
$$

Отметим теперь, что, поскольку $\mathbb{V} \hookrightarrow \mathbb{W}_{1}$ и $u_{m} \rightarrow u$ слабо в $\mathbb{V}$, для почти всех $t \in(0, T)$ следует, что для некоторой подпоследовательности последовательности $\left\{u_{m}\right\}$ имеем $u_{m} \rightarrow u$ сильно в $\mathbb{W}_{1}$ для почти всех $t \in(0, T)$. Отсюда и из условия $\mathbb{L}, 3)$ следует сильная сходимость $\mathbb{L} u_{m} \rightarrow \mathbb{L} u$ в $\mathbb{W}_{1}^{*}$ для почти всех $t \in(0, T)$. Нетрудно убедиться в том, что из условий $\mathbb{D P}$ вытекает сильная сходимость $\mathbb{D P}\left(u_{m}\right) \mapsto \mathbb{D P}(u)$ в $\mathbb{V}_{0}^{*}$ для почти всех $t \in(0, T)$.

Шаг 3. Метод монотонности. Воспользуемся теперь методом монотонности. Напомним, что на шаге 2 мы получили предельные включения (3.22) и (3.24) и, кроме того,

$$
\mathbb{A}\left(u_{m}\right) \rightarrow \chi \quad * \text {-слабо в } \mathbb{L}^{\infty}\left(0, T ; \mathbb{V}^{*}\right), \quad \mathbb{A}(u) \equiv \sum_{j=0}^{N} \mathbb{A}_{j}(u) .
$$

Далее потребуется следуюшая лемма.

Лемма 3.2. Пусть $\mathbb{A}(u) \equiv \sum_{j=0}^{N} \mathbb{A}_{j}(u)$. Тогда для некоторого $T>0$ найдется такая подпоследовательность последовательности $\left\{u_{m}\right\}$, что $\mathbb{A}\left(u_{m}\right) \rightarrow \mathbb{A}(u) \quad *$-слабо в $\mathbb{L}^{\infty}\left(0, T ; \mathbb{V}^{*}\right)$. Кроме того, для некоторой подпоследовательности последовательности $\left\{u_{m}\right\}$ выполнено свойство

$$
u_{m} \rightarrow u \quad \text { сильно в } \mathbb{L}^{p_{j}}\left(0, T ; \mathbb{V}_{j}\right), \quad j=\overline{0, N} .
$$

ДокАЗАТЕЛЬСтво. Перепишем уравнение (3.2) в эквивалентном виде:

$$
\left\langle\mathbb{A}\left(u_{m}\right), w_{j}\right\rangle=\left\langle\mathbb{A}\left(u_{m 0}\right), w_{j}\right\rangle+\int_{0}^{t}\left[\left(\mathbb{F}\left(u_{m}\right), w_{j}\right)_{0}-\left(\mathbb{L} u_{m}, w_{j}\right)_{1}-\left\langle\mathbb{D P}\left(u_{m}\right), w_{j}\right\rangle_{0}\right] d s,
$$

где $\langle\cdot, \cdot\rangle$ - скобки двойственности между банаховыми пространствами $\mathbb{V}$ и $\mathbb{V}^{*}$.

Зафиксируем $j \in \mathbb{N}$ и перейдем в уравнении (3.26) к пределу при $m \rightarrow+\infty$; тогда получим

$$
\chi=\int_{0}^{t}[\mathbb{F}(u)-\mathbb{L} u-\mathbb{D P}(u)] d s+\mathbb{A}\left(u_{0}\right)
$$

Пусть

$$
\begin{aligned}
0 \leqslant \mathbb{X}_{\mu} \equiv \int_{0}^{T}\left\langle\mathbb{A}\left(u_{\mu}\right)-\mathbb{A}(v), u_{\mu}-v\right\rangle d t \\
=\int_{0}^{T}\left\langle\mathbb{A}\left(u_{\mu}\right), u_{\mu}\right\rangle d t-\int_{0}^{T}\left\langle\mathbb{A}\left(u_{\mu}\right), v\right\rangle d t-\int_{0}^{T}\left\langle\mathbb{A}(v), u_{\mu}-v\right\rangle d t \\
\int_{0}^{T}\left\langle\mathbb{A}\left(u_{\mu}\right), u_{\mu}\right\rangle d t=\int_{0}^{T}\left\langle\mathbb{A}\left(u_{\mu 0}\right), u_{\mu}\right\rangle d t \\
+\int_{0}^{T} \int_{0}^{t}\left[\left(\mathbb{F}\left(u_{\mu}\right)(s), u_{\mu}(t)\right)_{0}-\left(\mathbb{L} u_{\mu}(s), u_{\mu}(t)\right)_{1}\right. \\
\left.-\left\langle\mathbb{D P}\left(u_{\mu}\right)(s), u_{\mu}(t)\right\rangle_{0}\right] d s d t
\end{aligned}
$$


Отсюда получим, что

$$
\begin{aligned}
0 \leqslant \limsup _{\mu \rightarrow+\infty} \mathbb{X}_{\mu} \leqslant & \int_{0}^{T} \int_{0}^{t}\left[(\mathbb{F}(u)(s), u(t))_{0}-(\mathbb{L} u(s), u(t))_{1}-\langle\mathbb{D P}(u)(s), u(t)\rangle_{0}\right] d s d t \\
& +\int_{0}^{T}\left\langle\mathbb{A}\left(u_{0}\right), u\right\rangle d t-\int_{0}^{T}\langle\chi, v\rangle d t-\int_{0}^{T}\langle\mathbb{A}(v), u-v\rangle d t \\
= & \int_{0}^{T}\langle\chi-\mathbb{A}(v), u-v\rangle d t \geqslant 0
\end{aligned}
$$

Положим теперь $v=u-\lambda w$ для любых $v, w \in \mathbb{L}^{r}(0, T ; \mathbb{V}), \quad \lambda>0, u \in$ $\mathbb{L}^{\infty}(0, T ; \mathbb{V}) \subset \mathbb{L}^{r}(0, T ; \mathbb{V}), \quad \chi, \mathbb{A}(v) \in \mathbb{L}^{r^{\prime}}\left(0, T ; \mathbb{V}^{*}\right), \quad r>1, \quad r^{\prime}=r /(r-1)$. Справедливо следуюшее неравенство:

$$
\int_{0}^{T}\langle\chi-\mathbb{A}(u-\lambda w), w\rangle d t \geqslant 0
$$

из которого в силу полунепрерьвности каждого из операторов $\mathbb{A}_{j}(v), j=\overline{0, N}$, получим $\chi=\mathbb{A}(u)$.

$\mathrm{C}$ другой стороны,

$$
\begin{aligned}
& \limsup _{\mu \rightarrow+\infty} \int_{0}^{T}\left\langle\mathbb{A}_{j}\left(u_{\mu}\right), u_{\mu}\right\rangle_{j} d t \leqslant-\liminf _{\mu \rightarrow+\infty} \sum_{k=0, k \neq j}^{N} \int_{0}^{T}\left\langle\mathbb{A}_{k}\left(u_{\mu}\right), u_{\mu}\right\rangle_{k} d t \\
& \quad+\limsup _{\mu \rightarrow+\infty} \int_{0}^{T}\left\langle\mathbb{A}\left(u_{\mu}\right), u_{\mu}\right\rangle d t \leqslant-\sum_{k=0, k \neq j}^{N} \int_{0}^{T}\left\langle\mathbb{A}_{k}(u), u\right\rangle_{k} d t+\int_{0}^{T}\langle\mathbb{A}(u), u\rangle d t \\
& \quad=\int_{0}^{T}\left\langle\mathbb{A}_{j}(u), u\right\rangle_{j} d t
\end{aligned}
$$

где мы воспользовались тем, что каждый из операторов $\mathbb{A}_{j}(v), j=\overline{0, N}$, в силу условий $\left.\mathbb{A}_{0}, 3^{*}\right)$ и $\left.\mathbb{A}, 3^{*}\right)$ порождает нормы рефлексивных банаховых пространств $\mathbb{L}^{2}\left(0, T ; \mathbb{V}_{0}\right)$ и $\mathbb{L}^{p_{j}}\left(0, T ; \mathbb{V}_{j}\right)$, эквивалентные исходным в силу условий $\left.\mathbb{A}_{0}, 3\right)$ и $\left.\mathbb{A}, 3\right)$, следующим образом:

$$
\left(\int_{0}^{T}\left\langle\mathbb{A}_{0} u, u\right\rangle_{0} d t\right)^{1 / 2}, \quad\left(\int_{0}^{T}\left\langle\mathbb{A}_{j}(u), u\right\rangle_{j} d t\right)^{1 / p_{j}}
$$

Наконец, в силу слабой сходимости $u_{\mu} \rightarrow u$ в $\mathbb{L}^{p_{j}}(0, T ; \mathbb{V}), j=\overline{0, N}$, и того, что операторы $\mathbb{A}_{j}(v), j=\overline{0, N}$, по указанным вьше правилам порождают нормы реффлексивных банаховых пространств, имеем

$$
\liminf _{m \rightarrow+\infty} \int_{0}^{T}\left\langle\mathbb{A}_{j}\left(u_{m}\right), u_{m}\right\rangle_{j} d t \geqslant \int_{0}^{T}\left\langle\mathbb{A}_{j}(u), u\right\rangle_{j} d t .
$$

Из соотношений $(3.29)$ и (3.30) следует, что

$$
\lim _{m \rightarrow+\infty} \int_{0}^{T}\left\langle\mathbb{A}_{j}\left(u_{m}\right), u_{m}\right\rangle_{j} d t=\int_{0}^{T}\left\langle\mathbb{A}_{j}(u), u\right\rangle_{j} d t
$$


Теперь в силу условия $\mathbb{A}, 3)$ выражения

$$
\left(\int_{0}^{T}\left\langle\mathbb{A}_{j}(u), u\right\rangle_{j} d t\right)^{1 / p_{j}}, \quad\left(\int_{0}^{T}\left\langle\mathbb{A}_{0} u, u\right\rangle_{0} d t\right)^{1 / 2}
$$

являются нормами, эквивалентными исходным нормам

$$
\left(\int_{0}^{T}\|u\|_{j}^{p_{j}} d t\right)^{1 / p_{j}}, \quad\left(\int_{0}^{T}\|u\|_{0}^{2} d t\right)^{1 / 2}
$$

банаховых пространств $\mathbb{L}^{p_{j}}\left(0, T ; \mathbb{V}_{j}\right)$. Поэтому из (3.31) следует сушествование такой подпоследовательности последовательности $\left\{u_{m}\right\}$, что

$$
\begin{aligned}
\left(\int_{0}^{T}\left\langle\mathbb{A}_{j}\left(u_{m}\right), u_{m}\right\rangle_{j} d t\right)^{1 / p_{j}} & \rightarrow\left(\int_{0}^{T}\left\langle\mathbb{A}_{j}(u), u\right\rangle_{j} d t\right)^{1 / p_{j}}, j=\overline{1, N} \\
\left(\int_{0}^{T}\left\langle\mathbb{A}_{0} u_{m}, u_{m}\right\rangle_{0} d t\right)^{1 / 2} & \rightarrow\left(\int_{0}^{T}\left\langle\mathbb{A}_{0} u, u\right\rangle_{0} d t\right)^{1 / 2}
\end{aligned}
$$

С другой стороны, имеет место слабая сходимость $u_{m} \rightarrow u$ в $\mathbb{L}^{p_{j}}\left(0, T ; \mathbb{V}_{j}\right)$, $j=\overline{0, N}$, значит, найдется такая подпоследовательность последовательности $\left\{u_{m}\right\}$, что $u_{m} \rightarrow u$ сильно сходится в каждом $\mathbb{L}^{p_{j}}\left(0, T ; \mathbb{V}_{j}\right), j=\overline{0, N}$. Лемма доказана.

Шаг 4. Предельный переход и единственность. Из предельных выражений $(3.22),(3.24)$ и (3.25) вытекает возможность перехода к пределу при $m \rightarrow+\infty$ в уравнении (3.26). В результате указанного предельного перехода в смысле $\mathbb{L}^{2}\left(0, T ; \mathbb{V}^{*}\right)$ получим

$$
\begin{gathered}
\left\langle\mathbb{A}_{0} u, v\right\rangle_{0}+\sum_{j=1}^{N}\left\langle\mathbb{A}_{j}(u), v\right\rangle_{j}=\left\langle\mathbb{A}_{0} u_{0}, v\right\rangle_{0}+\sum_{j=1}^{N}\left\langle\mathbb{A}_{j}\left(u_{0}\right), v\right\rangle_{j} \\
+\int_{0}^{t}\left[(\mathbb{F}(u), v)_{0}(s)-(\mathbb{L} u, v)_{1}(s)-\langle\mathbb{D P}(u), v\rangle_{0}\right] d s \quad \forall v \in \mathbb{V}, \\
u(0)=u_{0} \in \mathbb{V} .
\end{gathered}
$$

С другой стороны, $u^{\prime} \in \mathbb{L}^{2}\left(0, T ; \mathbb{V}_{0}\right), u \in \mathbb{L}^{\infty}(0, T)$. Перепишем равенство (3.32) в следуюшем виде:

$$
\begin{aligned}
\sum_{j=1}^{N}\left\langle\mathbb{A}_{j}(u), v\right\rangle_{j}= & -\left\langle\mathbb{A}_{0} u, v\right\rangle_{0}+\left\langle\mathbb{A}_{0} u_{0}, v\right\rangle_{0}+\sum_{j=1}^{N}\left\langle\mathbb{A}_{j}\left(u_{0}\right), v\right\rangle_{j} \\
& +\int_{0}^{t}\left[(\mathbb{F}(u), v)_{0}(s)-(\mathbb{L} u, v)_{1}(s)-\langle\mathbb{D P}(u), v\rangle_{0}\right] d s \quad \forall v \in \mathbb{V} .
\end{aligned}
$$


Правая часть данного равенства дифференцируема по $t \in(0, T)$ в смысле Лебега, значит, дифференцируема и левая часть, и мы приходим к выводу, что каждое решение уравнения (3.32) является решением уравнения

$$
\frac{d}{d t}\left\langle\mathbb{A}_{0} u, v\right\rangle_{0}+\frac{d}{d t} \sum_{j=1}^{N}\left\langle\mathbb{A}_{j}(u), v\right\rangle_{j}=(\mathbb{F}(u), v)_{0}-(\mathbb{L} u, v)_{1}-\langle\mathbb{D P}(u), v\rangle_{0} \quad \forall v \in \mathbb{V},
$$

понимаемого в смысле $\mathcal{D}^{\prime}(0, T)$. Перепишем теперь уравнение $(3.1)$ в виде

$$
\frac{d}{d t} \sum_{j=1}^{N}\left\langle\mathbb{A}_{j}(u), v\right\rangle_{j}=-\frac{d}{d t}\left\langle\mathbb{A}_{0} u, v\right\rangle_{0}+(\mathbb{F}(u), v)_{0}-(\mathbb{L} u, v)_{1}-\langle\mathbb{D P}(u), v\rangle_{0} \quad \forall v \in \mathbb{V},
$$

откуда из принадлежности $u \in \mathbb{L}^{\infty}(0, T ; \mathbb{V})$ приходим к выводу, что

$$
\sum_{j=1}^{N}\left\langle\mathbb{A}_{j}(u), v\right\rangle_{j} \in \mathbb{L}^{\infty}(0, T), \quad \frac{d}{d t} \sum_{j=1}^{N}\left\langle\mathbb{A}_{j}(u), v\right\rangle_{j} \in \mathbb{L}^{2}(0, T) \quad \forall v \in \mathbb{V} .
$$

В силу этого каждое решение $u$ задачи $(3.1)$ класса $\mathbb{L}^{\infty}(0, T ; \mathbb{V}), u^{\prime} \in \mathbb{L}^{2}\left(0, T ; \mathbb{V}_{0}\right)$, является решением уравнения (3.32). Значит, для функции $u \in \mathbb{L}^{\infty}(0, T ; \mathbb{V}), u^{\prime} \in$ $\mathbb{L}^{2}\left(0, T ; \mathbb{V}_{0}\right)$, задачи $(3.1)$ и (3.32) эквивалентны.

Для доказательства единственности решения рассматриваемой задачи воспользуемся эквивалентным к (3.1) уравнением (3.32); тогда получим уравнение

$$
\mathbb{A}_{0} u+\sum_{j=1}^{N} \mathbb{A}_{j}(u)=\int_{0}^{t}[\mathbb{F}(u)-\mathbb{L} u(s)-\mathbb{D P}(u)] d s+\mathbb{A}_{0} u_{0}+\sum_{j=1}^{N} \mathbb{A}_{j}\left(u_{0}\right),
$$

которое понимается в смысле $\mathbb{L}^{\infty}\left(0, T ; \mathbb{V}^{*}\right)$.

Пусть $u_{1}$ и $u_{2}-$ два решения уравнения $(3.33)$ класса $\mathbb{L}^{\infty}(0, T ; \mathbb{V})$ с одной и той же начальной функцией $u_{0} \in \mathbb{V}$. Тогда для разности этих решений $w=u_{1}-u_{2}$ получим соотношение

$$
\begin{gathered}
\left\langle\mathbb{A}_{0} w, w\right\rangle_{0}+\sum_{j=1}^{N}\left\langle\mathbb{A}_{j}\left(u_{1}\right)-\mathbb{A}_{j}\left(u_{2}\right), w\right\rangle=\int_{0}^{t}\left[\left(\mathbb{F}\left(u_{1}\right)(s)-\mathbb{F}\left(u_{2}\right)(s), w(t)\right)_{0}\right. \\
\left.-\left(\mathbb{L} u_{1}(s)-\mathbb{L} u_{2}(s), w(t)\right)_{1}-\left\langle\mathbb{D P}\left(u_{1}\right)-\mathbb{D P}\left(u_{2}\right), w\right\rangle_{0}\right] d s
\end{gathered}
$$

откуда в силу монотонности операторов $\mathbb{A}_{j}(u)$, а также с учетом условия $\left.\mathbb{A}_{0}, 2\right)$ и вложений $\mathbb{V}_{0} \subseteq \mathbb{W}_{0}$ и $\mathbb{V}_{0} \subseteq \mathbb{W}_{1}$ приходим к неравенству

$$
\begin{aligned}
\|w\|_{0}^{2}(t) \leqslant & C|w|_{0}(t) \int_{0}^{t}\left|\mathbb{F}\left(u_{1}\right)-\mathbb{F}\left(u_{2}\right)\right|_{0}^{*} d s+C|w|_{1}(t) \int_{0}^{t}\left|\mathbb{L} u_{1}-\mathbb{L} u_{2}\right|_{1}^{*} d s \\
& +C\|w\|_{0}(t) \int_{0}^{t}\left|\mathbb{D P}\left(u_{1}\right)-\mathbb{D P P}\left(u_{2}\right)\right|_{3} d s \\
\leqslant & C\|w\|_{0}(t) \int_{0}^{t}\|w\|_{0}(s) d s, \quad C>0
\end{aligned}
$$

из которого в силу теоремы Гронуолла-Белмана получаем, что $u_{1}=u_{2}$ почти всюду на множестве $(0, T) \times \Omega$. 
Шаг 5. Разрушение решений. Справедлива следуюшая лемма.

ЛЕмМа 3.3. Пусть

$$
\Phi \equiv \frac{1}{2}\left\langle\mathbb{A}_{0} u, u\right\rangle_{0}+\sum_{j=1}^{N} \frac{p_{j}-1}{p_{j}}\left\langle\mathbb{A}_{j}(u), u\right\rangle_{j}, \quad \alpha \equiv \frac{q+2}{\bar{p}}, \quad \bar{p} \equiv \max _{j=\overline{1, N}} p_{j}
$$

Тогда:

1) если $\alpha \in(0,1)$, то $T_{0}=+\infty$ и имеет место неравенство

$$
\Phi \leqslant \Phi_{0}\left[1+(1-\alpha) C_{2} \Phi_{0}^{\alpha-1} t\right]^{1 /(1-\alpha)}
$$

2) если $\alpha=1$, то $T_{0}=+\infty$ и имеет место неравенство $\Phi \leqslant \Phi_{0} \exp \left\{C_{2} t\right\}$;

3) если $\alpha>1$ и выполнень условия

$$
\Phi^{\prime}(0)>\frac{\beta}{\alpha-1} \Phi(0), \quad \Phi^{\prime}(0)>\sqrt{\frac{\gamma}{\alpha-1}} \Phi(0),
$$

то найдется такое $T_{0} \in\left[T_{1}, T_{2}\right]$, что справедливо соотношение

$$
\lim _{t \uparrow T_{0}} \Phi(t)=+\infty
$$

$2 \partial e$

$$
\begin{gathered}
T_{1}=\frac{2}{q} \Phi_{0}^{-q / 2} \bar{B}^{-1}, \quad T_{2}=\Phi_{0}^{1-\alpha} A^{-1} \\
C_{2} \equiv B C_{j^{*}}^{q+2}\left(\frac{\bar{p}}{\bar{p}-1}\right)^{\alpha}, \quad|v|_{0} \leqslant C_{j^{*}}\left\langle\mathbb{A}_{j^{*}} v, v\right\rangle_{j^{*}}^{1 / p_{j^{*}}} \\
\Phi_{0} \equiv \Phi(0), \quad \Phi^{\prime}(0) \equiv\left(\mathbb{F}\left(u_{0}\right), u_{0}\right)_{0}-\left(\mathbb{L} u_{0}, u_{0}\right)_{1} \\
\alpha \equiv \frac{\bar{p}+q+2}{2 \bar{p}}, \quad \beta \equiv \frac{(q+2)(q+1)}{q+2-\bar{p}} \\
\gamma \equiv \frac{4(q+1)^{2}}{q+2-\bar{p}}, \quad q+2>\bar{p} \equiv \max _{j=\overline{1, n}} p_{j} \\
A^{2} \equiv(\alpha-1)^{2} \Phi_{0}^{-2 \alpha}\left[\left(\Phi^{\prime}(0)\right)^{2}-\frac{\gamma}{\alpha-1} \Phi^{2}(0)\right] \\
\bar{B}=2^{q+2} B C_{1}^{q+2},
\end{gathered}
$$

$C_{1}$ - константа наилучшего вложения $\mathbb{V} \hookrightarrow \mathbb{W}_{0}, \quad B$ - константа из условия $\mathbb{F}, 4)$. 
ДоКАЗАТЕЛЬСТво. В силу условия $\mathbb{A}, 2$ ) справедливо неравенство Шварца [30] для производных $\Phi$ реше $\mathbb{A}_{j, u}^{\prime}: \mathbb{V}_{j} \rightarrow \mathcal{L}\left(\mathbb{V}_{j} ; \mathbb{V}_{j}^{*}\right)$ операторов $\mathbb{A}_{j}: \mathbb{V}_{j} \rightarrow \mathbb{V}_{j}^{*}$ :

$$
\begin{aligned}
\left|\left\langle\left(\mathbb{A}_{j}\left(u_{m}\right)\right)^{\prime}, u_{m}\right\rangle_{j}\right| & =\left|\left\langle\mathbb{A}_{j, u_{m}}^{\prime}\left(u_{m}\right) u_{m}^{\prime}, u_{m}\right\rangle_{j}\right| \\
& \leqslant\left\langle\mathbb{A}_{j, u_{m}}^{\prime}\left(u_{m}\right) u_{m}^{\prime}, u_{m}^{\prime}\right\rangle_{j}^{1 / 2}\left\langle\mathbb{A}_{j, u_{m}}^{\prime}\left(u_{m}\right) u_{m}, u_{m}\right\rangle_{j}^{1 / 2} \\
& =\left\langle\left(\mathbb{A}_{j}\left(u_{m}\right)\right)^{\prime}, u_{m}^{\prime}\right\rangle_{j}^{1 / 2}\left(p_{j}-1\right)^{1 / 2}\left\langle\mathbb{A}_{j}\left(u_{m}\right), u_{m}\right\rangle_{j}^{1 / 2}, \\
\left|\left\langle\mathbb{A}_{0} u_{m}^{\prime}, u_{m}\right\rangle_{0}\right| & \leqslant\left\langle\mathbb{A}_{0} u_{m}^{\prime}, u_{m}^{\prime}\right\rangle_{0}^{1 / 2}\left\langle\mathbb{A}_{0} u_{m}, u_{m}\right\rangle_{0}^{1 / 2} .
\end{aligned}
$$

Введем обозначение

$$
\Phi_{m} \equiv \frac{1}{2}\left\langle\mathbb{A}_{0} u_{m}, u_{m}\right\rangle_{0}+\sum_{j=1}^{N} \frac{p_{j}-1}{p_{j}}\left\langle\mathbb{A}_{j}\left(u_{m}\right), u_{m}\right\rangle_{j} .
$$

Из соотношений (3.34)-(3.36) вытекает

$$
\begin{aligned}
\left|\frac{d}{d t} \Phi_{m}\right|^{2} \leqslant & \left|\left\langle\mathbb{A}_{0} u_{m}^{\prime}, u_{m}\right\rangle_{0}\right|+\left.\sum_{j=1}^{N}\left|\left\langle\left(\mathbb{A}_{j}\left(u_{m}\right)\right)^{\prime}, u_{m}\right\rangle_{j}\right|\right|^{2} \\
\leqslant & \left(\left\langle\mathbb{A}_{0} u_{m}^{\prime}, u_{m}^{\prime}\right\rangle_{0}+\sum_{j=1}^{N}\left\langle\left(\mathbb{A}_{j}\left(u_{m}\right)\right)^{\prime}, u_{m}^{\prime}\right\rangle_{j}\right) \\
& \times\left(\left\langle\mathbb{A}_{0} u_{m}, u_{m}\right\rangle_{0}+\sum_{j=1}^{N}\left(p_{j}-1\right)\left\langle\mathbb{A}_{j}\left(u_{m}\right), u_{m}\right\rangle_{j}\right) \\
\leqslant & \bar{p}\left(\left\langle\mathbb{A}_{0} u_{m}^{\prime}, u_{m}^{\prime}\right\rangle_{0}+\sum_{j=1}^{N}\left\langle\left(\mathbb{A}_{j}\left(u_{m}\right)\right)^{\prime}, u_{m}^{\prime}\right\rangle_{j}\right) \\
& \times\left(\frac{1}{\bar{p}}\left\langle\mathbb{A}_{0} u_{m}, u_{m}\right\rangle_{0}+\sum_{j=1}^{N} \frac{p_{j}-1}{\bar{p}}\left\langle\mathbb{A}_{j}\left(u_{m}\right), u_{m}\right\rangle_{j}\right) \\
\leqslant & \bar{p}\left(\left\langle\mathbb{A}_{0} u_{m}^{\prime}, u_{m}^{\prime}\right\rangle_{0}+\sum_{j=1}^{N}\left\langle\left(\mathbb{A}_{j}\left(u_{m}\right)\right)^{\prime}, u_{m}^{\prime}\right\rangle_{j}\right) \\
& \times\left(\frac{1}{2}\left\langle\mathbb{A}_{0} u_{m}, u_{m}\right\rangle_{0}+\sum_{j=1}^{N} \frac{p_{j}-1}{p_{j}}\left\langle\mathbb{A}_{j}\left(u_{m}\right), u_{m}\right\rangle_{j}\right) \\
&
\end{aligned}
$$

где $\bar{p}=\max _{j=\overline{1, N}} p_{j}>2$.

Справедливо следующее утверждение.

УТВЕРЖДЕНИЕ 3.1. Пусть выполнены условия $\mathbb{L} u \mathbb{A}_{0} u$, кроме того, $\mathbb{V}_{0} \subset \mathbb{W}_{1}$. Тогда справедливо неравенство

$$
(\mathbb{L} u, u)_{1} \leqslant C\left\langle\mathbb{A}_{0} u, u\right\rangle_{0} \quad \forall u \in \mathbb{V}_{0}
$$

при некотором $C>0$. 
ДокАЗАТЕльСтво. Действительно, пусть $u \in \mathbb{V}_{0} \subset \mathbb{W}_{1}$; тогда справедлива следующая цепочка неравенств:

$$
(\mathbb{L} u, u)_{1} \leqslant D_{1}|u|_{1}^{2} \leqslant D_{1} C_{1}\|u\|_{0}^{2} \leqslant D_{1} C_{1} m_{0}^{-1}\left\langle\mathbb{A}_{0} u, u\right\rangle_{0}=C\left\langle\mathbb{A}_{0} u, u\right\rangle_{0} .
$$

Утверждение доказано.

Без ограничения общности можно считать постоянную $C$ в утверждении 3.1 равной единице. Действительно, введем новые операторы

$$
\widetilde{\mathbb{L}} \equiv C^{-1} \mathbb{L}, \quad \widetilde{\mathbb{F}} \equiv C^{-1} \mathbb{F}
$$

и, кроме того, сделаем замену $\tilde{t} \equiv C t$. Тогда для введенных операторов будет иметь место утверждение 3.1 с постоянной $C=1$. В силу утверждения 3.1 и неравенства Гёльдера справедливы неравенства

$$
\left(\mathbb{L} u_{m}, u_{m}^{\prime}\right)_{1} \leqslant\left(\mathbb{L} u_{m}, u_{m}\right)_{1}^{1 / 2}\left(\mathbb{L} u_{m}^{\prime}, u_{m}^{\prime}\right)_{1}^{1 / 2} \leqslant \frac{\varepsilon_{0}}{2}\left\langle\mathbb{A}_{0} u_{m}^{\prime}, u_{m}^{\prime}\right\rangle_{0}+\frac{1}{2 \varepsilon_{0}}\left\langle\mathbb{A}_{0} u_{m}, u_{m}\right\rangle_{0} .
$$

В силу утверждения 2.1 и неравенства Гёльдера справедливы неравенства

$$
\begin{aligned}
\left\langle\mathbb{D P}\left(u_{m}\right), u_{m}^{\prime}\right\rangle_{0} & \leqslant\left\langle\mathbb{A}_{0} u_{m}^{\prime}, u_{m}^{\prime}\right\rangle_{0}^{1 / 2}\left(\mathbb{F}\left(u_{m}\right), u_{m}\right)_{0}^{1 / 2} \\
& \leqslant \frac{\varepsilon_{0}}{2}\left\langle\mathbb{A}_{0} u_{m}^{\prime}, u_{m}^{\prime}\right\rangle_{0}+\frac{1}{2 \varepsilon_{0}}\left(\mathbb{F}\left(u_{m}\right), u_{m}\right)_{0} .
\end{aligned}
$$

Введем обозначение

$$
\mathbb{J}_{m} \equiv\left\langle\mathbb{A}_{0} u_{m}^{\prime}, u_{m}^{\prime}\right\rangle_{0}+\sum_{j=1}^{N}\left\langle\left(\mathbb{A}_{j}\left(u_{m}\right)\right)^{\prime}, u_{m}^{\prime}\right\rangle_{j} .
$$

Тогда из энергетических равенств (3.6), (3.10) с учетом неравенств (3.38) и (3.39) для функции $\mathbb{J}_{m}$ вытекает цепочка неравенств

$$
\begin{aligned}
\mathbb{J}_{m} \leqslant & -\left(\mathbb{L} u_{m}, u_{m}\right)_{1}+\frac{\varepsilon_{0}}{2}\left\langle\mathbb{A}_{0} u_{m}^{\prime}, u_{m}^{\prime}\right\rangle_{0} \\
& +\frac{1}{2 \varepsilon_{0}}\left(\mathbb{F}\left(u_{m}\right), u_{m}\right)_{0}+\frac{1}{q+2} \Phi_{m}^{\prime \prime}+\frac{2}{q+2}\left(\mathbb{L} u_{m}, u_{m}^{\prime}\right)_{1} \\
\leqslant & \frac{q}{q+2}\left(\mathbb{L} u_{m}, u_{m}^{\prime}\right)_{1}+\frac{\varepsilon_{0}}{2}\left\langle\mathbb{A}_{0} u_{m}^{\prime}, u_{m}^{\prime}\right\rangle_{0}+\frac{1}{2 \varepsilon_{0}}\left(\mathbb{F}\left(u_{m}\right), u_{m}\right)_{0}+\frac{1}{q+2} \Phi_{m}^{\prime \prime} \\
\leqslant & \frac{q}{q+2}\left(\mathbb{L} u_{m}, u_{m}^{\prime}\right)_{1}+\frac{\varepsilon_{0}}{2} \mathbb{J}_{m}+\frac{1}{q+2} \Phi_{m}^{\prime \prime}+\frac{1}{2 \varepsilon_{0}}\left(\mathbb{L} u_{m}, u_{m}\right)_{1}+\frac{1}{2 \varepsilon_{0}} \Phi_{m}^{\prime} \\
\leqslant & \frac{1}{q+2} \Phi_{m}^{\prime \prime}+\frac{1}{2 \varepsilon_{0}} \Phi_{m}^{\prime}+\frac{2}{\varepsilon_{0}} \frac{q+1}{q+2} \Phi_{m}+\frac{q+1}{q+2} \varepsilon_{0} \mathbb{J}_{m} .
\end{aligned}
$$

Из (3.40) вытекает следующее неравенство:

$$
\left[1-\frac{q+1}{q+2} \varepsilon_{0}\right] \mathbb{J}_{m} \leqslant \frac{1}{q+2} \Phi_{m}^{\prime \prime}+\frac{1}{2 \varepsilon_{0}} \Phi_{m}^{\prime}+\frac{2}{\varepsilon_{0}} \frac{q+1}{q+2} \Phi_{m} .
$$


Используя соотношения (3.37) и (3.41), получаем обыкновенное дифференциальное неравенство второго порядка

$$
\Phi_{m} \Phi_{m}^{\prime \prime}-\alpha\left(\Phi_{m}^{\prime}\right)^{2}+\beta \Phi_{m} \Phi_{m}^{\prime}+\gamma \Phi_{m}^{2} \geqslant 0
$$

где

$$
\alpha \equiv \frac{q+2}{\bar{p}}\left[1-\frac{q+1}{q+2} \varepsilon_{0}\right], \quad \beta=\frac{q+2}{2 \varepsilon_{0}}, \quad \gamma=\frac{2 q+2}{\varepsilon_{0}} .
$$

Предположим, что $\varepsilon_{0} \in\left(0,(q+2-\bar{p})(q+1)^{-1}\right)$; тогда $\alpha>1$. Рассмотрим теперь детально неравенство (3.42). Данное неравенство нетрудно привести к линейному дифференциальному неравенству

$$
\mathbb{Z}_{m}^{\prime \prime}+\beta \mathbb{Z}_{m}^{\prime}-\delta \mathbb{Z}_{m} \leqslant 0, \quad \delta=\gamma(\alpha-1),
$$

относительно функции

$$
\mathbb{Z}_{m}=\Phi_{m}^{1-\alpha} .
$$

Перейдем теперь к новой функции

$$
\mathbb{Y}_{m} \equiv \mathbb{Z}_{m} e^{\beta t}
$$

в уравнении (3.43); тогда получим неравенство

$$
\mathbb{Y}_{m}^{\prime \prime}-\beta \mathbb{Y}_{m}^{\prime}-\delta \mathbb{Y}_{m} \leqslant 0
$$

Из соотношения (3.45) следует, что

$$
\mathbb{Y}_{m}^{\prime}=e^{\beta t}(\alpha-1) \Phi_{m}^{-\alpha}\left(-\Phi_{m}^{\prime}+\frac{\beta}{\alpha-1} \Phi_{m}\right)
$$

Предположим, что выполнены условия

$$
\Phi^{\prime}(0)>\frac{\beta}{\alpha-1} \Phi(0), \quad \Phi(t) \equiv \frac{1}{2}\left\langle\mathbb{A}_{0} u, u\right\rangle_{0}+\sum_{j=1}^{N} \frac{p_{j}-1}{p_{j}}\left\langle\mathbb{A}_{j}(u), u\right\rangle_{j} .
$$

Тогда, переходя, если необходимо, к подпоследовательности, получим

$$
\Phi_{m}^{\prime}(0)>\frac{\beta}{\alpha-1} \Phi_{m}(0) .
$$

В силу непрерывной дифференцируемости функции $\mathbb{Y}_{m}$, учитьвая условие (3.49) и соотношение (3.47), приходим к выводу, что найдется такой интервал $\left[0, T_{3 m}\right)$, принадлежащий области определения $\Phi_{m}(t)$, что $\mathbb{Y}_{m}^{\prime}<0$, причем если $T_{3 m} \leqslant T_{m 0}$, то $\mathbb{Y}_{m}^{\prime}=0$ при $t=T_{3 m}$. Следовательно, при $t \in\left[0, T_{2 m}\right]$ имеем $-\beta \mathbb{Y}_{m}^{\prime}(t)>0$. Отсюда и из неравенства (3.46) получим следующее дифференциальное неравенство:

$$
\mathbb{Y}_{m}^{\prime \prime}-\delta \mathbb{Y}_{m} \leqslant 0, \quad t \in\left[0, T_{3 m}\right]
$$


Предположим, что выполнено неравенство

$$
\Phi^{\prime}(0)>\frac{\sqrt{\gamma}}{\sqrt{\alpha-1}} \Phi(0)
$$

Тогда, переходя, если необходимо, к подпоследовательности, получим

$$
\Phi_{m}^{\prime}(0)>\frac{\sqrt{\gamma}}{\sqrt{\alpha-1}} \Phi_{m}(0)
$$

откуда вытекает неравенство

$$
\left(\mathbb{Y}_{m}^{\prime}\right)^{2} \geqslant \delta \mathbb{Y}_{m}^{2}+A_{m}^{2}
$$

где

$$
A_{m}^{2} \equiv(1-\alpha)^{2} \Phi_{m}^{-2 \alpha}(0)\left[\left(\Phi_{m}^{\prime}(0)\right)^{2}-\frac{\delta}{(1-\alpha)^{2}}\left(\Phi_{m}(0)\right)^{2}\right]>0 .
$$

Из неравенства (3.53) получим

$$
\mathbb{Y}_{m}^{\prime}(t) \leqslant-A_{m} \quad \forall t \in\left[0, T_{3 m}\right]
$$

Используя (3.55), получим, что на области определения функции $\Phi_{m}(t)$ выполнено неравенство $\mathbb{Y}_{m}^{\prime}<0$, а отсюда и из $(3.55)$ имеем

$$
\mathbb{Y}_{m}(t) \leqslant \mathbb{Y}_{m 0}-A_{m} t
$$

Значит, найдется такой момент времени $T_{m 0} \in\left(0, T_{2 m}\right)$, что

$$
\limsup _{t \uparrow T_{m 0}} \Phi_{m}(t)=+\infty
$$

где $T_{2 m}=\mathbb{Y}_{m}(0) A_{m}^{-1}$.

Рассмотрим отдельно условия (3.48) и (3.51). Заметим, что $\varepsilon_{0}$ выбрано произвольно из интервала $\left(0,(q+2-\bar{p})(q+1)^{-1}\right)$. Теперь в качестве $\varepsilon_{0}$ выберем точку минимума функций

$$
f_{1}=\frac{\beta}{\alpha-1}, \quad f_{2}=\sqrt{\frac{\gamma}{\alpha-1}}
$$

Нетрудно убедиться в том, что минимум достигается в точке

$$
\varepsilon_{0}=\frac{q+2-\bar{p}}{2 q+2}
$$

Из неравенства (3.42) вытекает оценка снизу для функции $\Phi_{m}(t)$ :

$$
\Phi_{m} \geqslant \frac{\Psi_{m 0} e^{-\theta t}}{\left[T_{2 m}-t\right]^{1 /(\alpha-1)}}
$$

где $T_{2 m} \equiv \Phi_{m 0}^{1-\alpha} A_{m}^{-1}, \quad \Psi_{m 0}=A_{m}^{1 /(1-\alpha)}, \quad \theta=\beta /(\alpha-1)$. 
Докажем теперь, что $\Phi_{m}(t) \rightarrow \Phi(t)$ для почти всех $t \in(0, T)$, где

$$
\Phi(t) \equiv \frac{1}{2}\left\langle\mathbb{A}_{0} u, u\right\rangle_{0}+\sum_{j=1}^{N} \frac{p_{j}-1}{p_{j}}\left\langle\mathbb{A}_{j}(u), u\right\rangle_{j}
$$

С одной стороны, вследствие сильной сходимости $u_{m} \rightarrow u$ в $\mathbb{L}^{p_{j}}\left(0, T ; \mathbb{V}_{j}\right)$ имеем

$$
\int_{0}^{t} \Phi_{m}(s) d s \rightarrow \int_{0}^{t} \Phi(s) d s
$$

С другой стороны, из (3.38) получаем

$$
\Phi_{m}=\Phi_{m 0}+\int_{0}^{t}\left[\left(\mathbb{F}\left(u_{m}\right), u_{m}\right)_{0}(s)-\left(\mathbb{L} u_{m}, u_{m}\right)_{1}(s)\right] d s
$$

По условию $\Phi_{m 0} \rightarrow \Phi_{0}, u_{m}(t) \rightarrow u(t)$ сильно в $\mathbb{W}_{0}$ и в $\mathbb{W}_{1}$ для почти всех $t \in(0, T)$, поэтому в силу ограниченной непрерывности по Липшицу оператора $\mathbb{F}(u): \mathbb{W}_{0} \rightarrow \mathbb{W}_{0}^{*}$ и непрерывности по Липшицу оператора $\mathbb{L}: \mathbb{W}_{1} \rightarrow \mathbb{W}_{1}^{*}$ приходим к выводу, что $\mathbb{F}\left(u_{m}\right) \rightarrow \mathbb{F}(u)$ сильно в $\mathbb{W}_{0}^{*}$ и $\mathbb{L} u_{m} \rightarrow \mathbb{L} u$ в $\mathbb{W}_{1}^{*}$ для почти всех $t \in(0, T)$. Понятно, что

$$
\left|\left(\mathbb{F}\left(u_{m}\right), u_{m}\right)_{0}\right| \leqslant B\left|u_{m}\right|_{0}^{q+2} \leqslant C\left\|u_{m}\right\|_{\mathbb{V}}^{q+2} \leqslant C,
$$

где $C$ не зависит от $m \in \mathbb{N}$ и от $t \in(0, T)$, а также

$$
\left(\mathbb{L} u_{m}, u_{m}\right)_{1} \leqslant D_{1}\left|u_{m}\right|_{1}^{2} \leqslant C\left\|u_{m}\right\|_{\mathbb{V}}^{2} \leqslant C,
$$

где $C$ не зависит от $m \in \mathbb{N}$ и от $t \in(0, T)$. Поэтому по теореме Лебега [31] получим, что для почти всех $t \in(0, T)$ последовательность функций $\Phi_{m}(t)$ почти всюду сходится к $\eta(t)$. Снова по теореме Лебега получим

$$
\int_{0}^{t} \Phi_{m} d s \rightarrow \int_{0}^{t} \eta d s
$$

Значит,

$$
\int_{0}^{t}[\Phi(s)-\eta(s)] d s=0 \quad \forall t \in(0, T),
$$

отсюда сразу получаем

$$
\Phi_{m}(t) \rightarrow \Phi(t)
$$

при почти всех $t \in(0, T)$. 
С учетом того, что $u_{m 0} \rightarrow u_{0}$ сильно в $\mathbb{V}$, при $m \rightarrow+\infty$ имеем

$$
\begin{aligned}
& \Phi_{m 0} \rightarrow \Phi_{0} \equiv \frac{1}{2}\left\langle\mathbb{A}_{0} u_{0}, u_{0}\right\rangle_{0}+\sum_{j=1}^{N} \frac{p_{j}-1}{p_{j}}\left\langle\mathbb{A}_{j}\left(u_{0}\right), u_{0}\right\rangle_{j} \\
& \Psi_{m 0} \rightarrow \Psi_{0} \equiv A_{0}^{1 /\left(\alpha_{1}-1\right)} .
\end{aligned}
$$

Из (3.58), переходя к пределу при $m \rightarrow+\infty$ в неравенствах (3.18), (3.19), получим

$$
\begin{aligned}
& \Phi(t) \leqslant \Phi_{0} \exp \left\{C_{2} t\right\}, \quad \alpha=\frac{q+2}{\bar{p}}=1, \\
& \Phi(t) \leqslant \Phi_{0}\left[1+(1-\alpha) C_{2} \Phi_{0}^{\alpha-1} t\right]^{1 /(1-\alpha)}, \quad \alpha<1,
\end{aligned}
$$

где

$$
\begin{gathered}
\alpha \equiv \frac{q+2}{\bar{p}}, \quad C_{2} \equiv B C_{j^{*}}^{q+2}\left(\frac{\bar{p}}{\bar{p}-1}\right)^{(q+2) / \bar{p}}, \\
|v|_{0} \leqslant C_{j^{*}}\left\langle\mathbb{A}_{j^{*}}(v), v\right\rangle_{j^{*}}^{1 / p_{j^{*}}}, \quad \bar{p}=\max _{j=\overline{1, N}} p_{j} .
\end{gathered}
$$

Рассмотрим теперь неравенства (3.13) и (3.57). Отметим, что при $m \rightarrow+\infty$

$$
\begin{gathered}
\Psi_{m 0} \rightarrow \Psi_{0} \equiv A^{1 /\left(\alpha_{1}-1\right)}, \quad T_{2 m} \rightarrow T_{2} \equiv \Phi_{0}^{1-\alpha_{1}} A^{-1}, \\
T_{1 m} \rightarrow T_{1}=\frac{2}{q} \Phi_{0}^{-q / 2} \bar{B}^{-1}, \\
\bar{B} \equiv 2^{q+2} B C_{1}^{q+2},
\end{gathered}
$$

где $C_{1}$ - константа наилучшего вложения $\mathbb{V} \hookrightarrow \mathbb{W}_{0}, B$ - константа из условия $\left.\mathbb{F}, 4\right)$.

Рассмотрим сначала неравенство (3.57). Без ограничения обшности переходом к подпоследовательности мы можем получить, что последовательность $T_{2 m}>0$ равномерно по $m$, поскольку $T_{2}>0$. В силу сходимости последовательности $T_{2 m} \rightarrow T_{2}$ при $m \rightarrow+\infty$ можно выбрать монотонно сходящуюся подпоследовательность, которую мы снова обозначим через $T_{2 m}$. Соответствуюшие подпоследовательности последовательностей $\left\{u_{0 m}\right\}$ и $\left\{u_{m}\right\}$ мы снова будем обозначать $\left\{u_{0 m}\right\}$ и $\left\{u_{m}\right\}$. Пусть $T_{2 m} \uparrow T_{2}$; тогда неравенство (3.43) имеет место равномерно по $t \in\left[0, T_{2} \bar{m}\right), m \geqslant \bar{m}$ для некоторого фиксированного $\bar{m} \in \mathbb{N}$. Переходя к пределу в неравенстве (3.57) при $m \rightarrow+\infty$, для таких $t$ получим

$$
\Phi \geqslant \frac{\Psi_{0} e^{-\theta t}}{\left[T_{2}-t\right]^{1 /(\alpha-1)}}, \quad \theta=\frac{\beta}{\alpha-1},
$$

при $t \in\left[0, T_{2} \bar{m}\right)$. Отсюда в силу произвольности $\bar{m} \in \mathbb{N}$ сразу же получаем, что (3.61) справедливо для всех $t \in\left[0, T_{2}\right)$ и время разрушения решения задачи (3.1) удовлетворяет оценке $T_{0} \leqslant T_{2}$. 
Пусть теперь $T_{2 m} \downarrow T_{2}$. Предположим, что $T_{0}>T_{2}$, и пусть, кроме того, $M \equiv \sup _{t \in\left[0, T_{2}\right]} \Phi(t)<+\infty$. Тогда неравенство (3.57) имеет место равномерно по $t \in\left[0, T_{2}\right)$. Переходя к пределу при $m \rightarrow+\infty$ в неравенстве (3.57), получим в силу сделанного предположения относительно $T_{0}$ следующее неравенство:

$$
\Phi \geqslant \frac{\Psi_{0} e^{-\theta t}}{\left[T_{2}-t\right]^{1 /(\alpha-1)}} \leqslant M<+\infty \text { при } t \in\left[0, T_{2}\right), \quad \theta=\frac{\beta}{\alpha-1} .
$$

Однако из полученного неравенства следует, что наше предположение не выполняется. Отсюда сразу следует, что $T_{0} \leqslant T_{2}$. Аналогичным образом из неравенства (3.13) вытекает, что

$$
\Phi(t) \leqslant \frac{\Phi_{0}}{\left[1-\frac{q}{2} \Phi_{0}^{q / 2} \bar{B} t\right]^{2 / q}} \quad \forall t \in\left(0, T_{1}\right), \quad T_{0} \geqslant T_{1} .
$$

Лемма доказана. Тем самым доказана и теорема 3.1 .

§4. Сильная обобщенная разрешимость, единственность и разрушение решения задачи (1.1)

ОПРЕДЕЛЕНИЕ 4.1. Сильным обобщенным решением задачи (1.1) называется решение класса $\mathbb{C}^{1}\left([0, T] ; \mathbb{V}_{0}\right)$, удовлетворяюшее условиям

$$
\begin{gathered}
\left\langle\left(\mathbb{A}_{0} u\right)^{\prime}, v\right\rangle_{0}+\sum_{j=1}^{N}\left\langle\left(\mathbb{A}_{j}(u)\right)^{\prime}, v\right\rangle_{j}+\langle\mathbb{D P}(u), v\rangle_{0}+(\mathbb{L} u, v)_{1}=(\mathbb{F}(u), v)_{0} \\
\forall v \in \mathbb{V}_{0}, \quad t \in[0, T] \\
u(0)=u_{0} \in \mathbb{V}_{0},
\end{gathered}
$$

где производная по времени понимается в классическом смысле.

В предположении, что производная Фреше $\mathbb{A}_{j, u}^{\prime}(u): \mathbb{V}_{j} \rightarrow \mathcal{L}\left(\mathbb{V}_{j} ; \mathbb{V}_{j}^{*}\right)$ оператора $\mathbb{A}_{j}$ является сильно непрерывной по $u \in \mathbb{V}_{j}$, получаем, что в рассматриваемом классе имеют место включения $\mathbb{A}_{0} u \in \mathbb{C}^{1}\left([0, T] ; \mathbb{V}_{0}^{*}\right), \mathbb{A}_{j}(u) \in \mathbb{C}^{1}\left([0, T] ; \mathbb{V}_{j}^{*}\right)$, $\mathbb{D P}(u) \in \mathbb{C}\left([0, T] ; \mathbb{W}_{4}\right), \quad \mathbb{L} u \in \mathbb{C}\left([0, T] ; \mathbb{W}_{1}^{*}\right), \mathbb{F}(u) \in \mathbb{C}\left([0, T] ; \mathbb{W}_{0}^{*}\right)$ и операторы $\mathbb{A}_{0} u$ и $\mathbb{A}_{j}(u)$ принадлежат классу $\mathbb{C}^{1}\left([0, T] ; \mathbb{V}_{0}^{*}\right)$, а $\mathbb{D P}(u), \mathbb{L} u, \mathbb{F}(u)$ - классу $\mathbb{C}\left([0, T] ; \mathbb{V}_{0}^{*}\right)$. Тем самым задача в силу условий следующей ниже теоремы 4.1 эквивалентна задаче

$$
\begin{gathered}
\left\langle\left(\mathbb{A}_{0} u\right)^{\prime}, v\right\rangle_{0}+\sum_{j=1}^{N}\left\langle\left(\mathbb{A}_{j}(u)\right)^{\prime}, v\right\rangle_{0}+\langle\mathbb{D P}(u), v\rangle_{0}+\langle\mathbb{L} u, v\rangle_{0}=\langle\mathbb{F}(u), v\rangle_{0} \\
\forall v \in \mathbb{V}_{0}, \quad t \in[0, T] \\
u(0)=u_{0} \in \mathbb{V}_{0},
\end{gathered}
$$

где производная по времени понимается в классическом смысле.

Справедлива следуюшая теорема. 
Теорема 4.1. Пусть выполнены условия $\mathbb{A}, \mathbb{A}_{0}, \mathbb{F}, \mathbb{L}, \mathbb{D P}$. Предположим, что $\mathbb{V}_{0} \subseteq \mathbb{V}_{j}, \quad j=\overline{1, N}, \quad \mathbb{V}_{0} \subseteq \mathbb{W}_{0} \subseteq \mathbb{W}_{2}, \quad \mathbb{V}_{0} \subseteq \mathbb{W}_{1}$, и пусть найдется $j^{*} \in \overline{1, N}$, при котором $\bar{p} \equiv p_{j^{*}} \geqslant p_{j}$ u $p_{j^{*}} \geqslant q+2, j=\overline{1, N}$, такое, что $\mathbb{V}_{j^{*}} \subseteq \mathbb{W}_{0}$. Пусть производнье Фреше

$$
\mathbb{A}_{j, u}^{\prime} \in \mathbb{B} \mathbb{C}\left(\mathbb{V}_{j} ; \mathcal{L}\left(\mathbb{V}_{j}, \mathbb{V}_{j}^{*}\right)\right)
$$

операторов $\mathbb{A}_{j}: \mathbb{V}_{j} \rightarrow \mathbb{V}_{j}^{*}$ являются ограниченными, сильно непрерывными и, кроме того, монотонными в том смысле, что

$$
\left\langle\mathbb{A}_{j, u}^{\prime}(u) u_{1}-\mathbb{A}_{j, u}^{\prime}(u) u_{2}, u_{1}-u_{2}\right\rangle_{j} \geqslant 0 \quad \forall u, u_{1}, u_{2} \in \mathbb{V}_{j}, \quad j=\overline{1, N} .
$$

Пусть выполнено неравенство $\left(\mathbb{F}\left(u_{0}\right), u_{0}\right)_{0}>0$. Тогда для любого $u_{0} \in \mathbb{V}_{0}$ найдется такое максимальное $T_{0} \equiv T_{u_{0}}>0$, что для любого $0<T<T_{0}$ задача Кочи (1.1) имеет точно одно непродолжаемое во времени решение u(t) класса $\mathbb{C}^{1}\left([0, T] ; \mathbb{V}_{0}\right)$, т. е. либо $T_{0}=+\infty$, либо $T_{0}<+\infty$, и в последнем случае справедливо предельное равенство

$$
\lim _{t \uparrow T_{0}}\|u\|_{0}=+\infty
$$

причем справедливы следующие оченки для разных значений $\alpha=(q+2) / \bar{p}$, $\bar{p}=\max _{j=\overline{1, n}} p_{j}$ :

1) если $\alpha \in(0,1)$, то $T_{0}=+\infty$, и тогда

$$
\Phi(t) \leqslant\left[\Phi_{0}^{1-\alpha}+(1-\alpha) \bar{A} t\right]^{1 /(1-\alpha)} ;
$$

2) если $\alpha=1$, mо $T_{0}=+\infty$, и тогда $\Phi(t) \leqslant \Phi_{0} \exp \{\bar{A} t\}$;

3) если $\alpha>1$ и выполнены условия

$$
\Phi^{\prime}(0)>\frac{\beta}{\alpha-1} \Phi(0), \quad \Phi^{\prime}(0)>\sqrt{\frac{\gamma}{\alpha-1}} \Phi(0),
$$

то $T_{0} \in\left[T_{1}, T_{2}\right]$ и справедливо соотношение

$$
\limsup _{t \uparrow T_{0}} \Phi(t)=+\infty
$$

२де

$$
\begin{gathered}
\Phi(t) \equiv \frac{1}{2}\left\langle\mathbb{A}_{0} u, u\right\rangle_{0}+\sum_{j=1}^{N} \frac{p_{j}-1}{p_{j}}\left\langle\mathbb{A}_{j}(u), u\right\rangle_{j}, \quad \Phi_{0} \equiv \Phi(0), \\
T_{1}=\frac{2}{q} \Phi_{0}^{-q / 2} \bar{B}^{-1}, \quad T_{2}=\Phi_{0}^{1-\alpha} A^{-1}, \quad \bar{B}=2^{(q+2) / 2} B C_{1}^{q+2}, \\
\bar{A} \equiv C_{j^{*}}^{q+2} M\left(\frac{\bar{p}}{\bar{p}-1}\right)^{(q+2) / \bar{p}}, \quad|v|_{0} \leqslant C_{j^{*}}\left\langle\mathbb{A}_{j^{*}} v, v\right\rangle_{j^{*}}^{1 / p_{j^{*}}}, \\
\Phi_{0} \equiv \Phi(0), \quad \Phi^{\prime}(0) \equiv\left(\mathbb{F}\left(u_{0}\right), u_{0}\right)_{0}-\left(\mathbb{L} u_{0}, u_{0}\right)_{1}, \\
\alpha \equiv \frac{\bar{p}+q+2}{2 \bar{p}}, \quad \beta \equiv \frac{(q+2)(q+1)}{q+2-\bar{p}}, \quad \gamma \equiv \frac{4(q+1)^{2}}{q+2-\bar{p}}, \quad q+2>\bar{p} \equiv \max _{j=1, n} p_{j}, \\
A^{2} \equiv(\alpha-1)^{2} \Phi_{0}^{-2 \alpha}\left[\left(\Phi^{\prime}(0)\right)^{2}-\frac{\gamma}{\alpha-1} \Phi^{2}(0)\right],
\end{gathered}
$$

$C_{1}$ - константа наилучшего вложения $\mathbb{V} \hookrightarrow \mathbb{W}_{0}, \quad B$ - константа из условия $\mathbb{F}, 4)$. 
ДокАЗАТЕльство. Доказательство этой теоремы также проведем в несколько этапов.

Шаг 1. Локальная разрешимость. Справедлива следуюшая лемма.

Лемма 4.1. Для любого $u_{0} \in \mathbb{V}_{0}$ найдется такое максимальное $T_{0}>0$, что существует единственное максимальное решение класса $\mathbb{C}^{1}\left([0, T] ; \mathbb{V}_{0}\right)$ для любого $T \in\left(0, T_{0}\right)$ задачи (1.1).

ДокАЗАтЕльство. Рассмотрим задачу (1.1). Заметим, что для оператора

$$
\mathbb{A}(u) \equiv \mathbb{A}_{0} u+\sum_{j=1}^{N} \mathbb{A}_{j}(u): \mathbb{V}_{0} \rightarrow \mathbb{V}_{0}^{*}
$$

справедливо равенство

$$
\begin{aligned}
\left\langle\mathbb{A}\left(u_{1}\right)-\mathbb{A}\left(u_{2}\right), u_{1}-u_{2}\right\rangle= & \left\langle\mathbb{A}_{0} u_{1}-\mathbb{A}_{0} u_{2}, u_{1}-u_{2}\right\rangle_{0} \\
& +\sum_{j=1}^{N}\left\langle\mathbb{A}_{j}\left(u_{1}\right)-\mathbb{A}_{j}\left(u_{2}\right), u_{1}-u_{2}\right\rangle \\
\geqslant & \left\langle\mathbb{A}_{0} u_{1}-\mathbb{A}_{0} u_{2}, u_{1}-u_{2}\right\rangle_{0}=\left\|u_{1}-u_{2}\right\|_{0}^{2} .
\end{aligned}
$$

Следовательно, для оператора $\mathbb{A}$ существует непрерьвный по Липшицу обратньй оператор $\mathbb{A}^{-1}: \mathbb{V}_{0}^{*} \rightarrow \mathbb{V}_{0}$ с постоянной Липшица, равной единице. С учетом этого сделаем замену $v=\mathbb{A}(u)$ в уравнении (1.1); тогда уравнение примет эквивалентный вид

$$
\frac{d v}{d t}=\mathbb{F}\left(\mathbb{A}^{-1}(v)\right)-\mathbb{L}^{-1}(v)-\mathbb{D P}\left(\mathbb{A}^{-1}(v)\right), \quad v(0)=v_{0}
$$

где $v_{0}=\mathbb{A}\left(u_{0}\right)$. Данная задача эквивалентна следующей задаче:

$$
v(t)=v_{0}+\int_{0}^{t}\left[\mathbb{F}\left(\mathbb{A}^{-1}(v)\right)-\mathbb{L} \mathbb{A}^{-1}(v)-\mathbb{D P}\left(\mathbb{A}^{-1}(v)\right)\right] d s .
$$

Воспользуемся методом сжимающих отображений для доказательства локальной разрешимости уравнения (4.2) в классе $\mathbb{C}^{1}\left([0, T] ; \mathbb{V}_{0}^{*}\right)$. С этой целью введем замкнутое ограниченное вьпуклое подмножество банахова пространства $\mathbb{L}^{\infty}\left(0, T ; \mathbb{V}_{0}^{*}\right)$ :

$$
\mathbb{B}_{R}=\left\{v \in \mathbb{L}^{\infty}\left(0, T ; \mathbb{V}_{0}^{*}\right):\|v\|_{T}^{*} \leqslant R\right\}
$$

где $\|v\|_{T}^{*}=$ ess. $\sup _{t \in(0, T)}\|v(t)\|_{0}^{*}$.

Докажем, что оператор

$$
\mathbb{H}(u) \equiv \int_{0}^{t}\left[\mathbb{F}\left(\mathbb{A}^{-1}(v)\right)-\mathbb{L} \mathbb{A}^{-1}(v)-\mathbb{D P}\left(\mathbb{A}^{-1}(v)\right)\right] d s
$$


действует из $\mathbb{B}_{R}$ в $\mathbb{B}_{R}$ и является сжимающим. Нетрудно доказать, что оператор $\mathbb{H}(v)$ действует из $\mathbb{B}_{R}$ в $\mathbb{B}_{R}$ при достаточно большом $R$ и при достаточно малом $T$. Поэтому рассмотрим вопрос о сжимаемости оператора $\mathbb{H}(v)$. Действительно, справедлива следующее неравенство:

$$
\begin{aligned}
\| \mathbb{H}\left(v_{1}\right) & -\mathbb{H}\left(v_{2}\right)\left\|_{T}^{*} \leqslant T \mu_{1}(\bar{R})\right\| \mathbb{A}^{-1}\left(v_{1}\right)-\mathbb{A}^{-1}\left(v_{2}\right) \|_{T} \\
& +T \mu_{2}(\bar{R})\left\|\mathbb{A}^{-1}\left(v_{1}\right)-\mathbb{A}^{-1}\left(v_{2}\right)\right\|_{T}+T C\left\|\mathbb{A}^{-1}\left(v_{1}\right)-\mathbb{A}^{-1}\left(v_{2}\right)\right\|_{T}
\end{aligned}
$$

где

$$
\|\cdot\|_{T} \equiv \underset{t \in(0, T)}{\operatorname{ess.} \sup }\|\cdot\|_{0}, \quad \bar{R}=\max \left(\left\|\mathbb{A}^{-1}\left(v_{1}\right)\right\|_{T},\left\|\mathbb{A}^{-1}\left(v_{2}\right)\right\|_{T}\right) .
$$

Из неравенства (4.3) в силу свойств оператора $\mathbb{A}^{-1}$ вытекает следующее неравенство:

$$
\left\|\mathbb{H}\left(v_{1}\right)-\mathbb{H}\left(v_{2}\right)\right\|_{T}^{*} \leqslant T C\left[1+\mu_{1}(R)+\mu_{2}(R)\right]\left\|v_{1}-v_{2}\right\|_{T}^{*} .
$$

При условии $T \leqslant \frac{1}{2}\left[1+\mu_{1}(R)+\mu_{2}(M)\right]^{-1} C^{-1}$ из неравенства (4.4) вытекает сжимаемость оператора $\mathbb{H}(v)$ на множестве $\mathbb{B}_{R}$. Значит, существует единственное решение $v$ уравнения (4.2) класса $\mathbb{L}^{\infty}\left(0, T ; \mathbb{V}_{0}^{*}\right)$. Используя стандартный алгоритм продолжения решений интегральных уравнений по времени, приходим к выводу, что найдется максимальное $T_{0}>0$, при котором сушествует единственное решение $v$ уравнения $(4.2)$ класса $\mathbb{L}^{\infty}\left(0, T_{0} ; \mathbb{V}_{0}^{*}\right)$, причем либо $T_{0}=+\infty$, либо $T_{0}<+\infty$, и в последнем случае имеет место предельное равенство

$$
\limsup _{t \rightarrow T_{0}}\|v(t)\|_{0}^{*}=+\infty
$$

Наконец, из уравнения (4.2) в силу сглаживающих свойств оператора $\mathbb{H}(v)$ получим, что $v \in \mathbb{C}^{1}\left(\left[0, T_{0}\right) ; \mathbb{V}_{0}^{*}\right)$.

Теперь заметим, что имеет место равенство

$$
\mathbb{A}(u) \equiv \mathbb{A}_{0} u+\sum_{j=1}^{N} \mathbb{A}_{j}(u)=v(x, t) \in \mathbb{C}^{1}\left(\left[0, T_{0}\right) ; \mathbb{V}_{0}^{*}\right)
$$

Уравнение $(4.5)$ для функции $u \in \mathbb{C}^{1}\left(\left[0, T_{0}\right) ; \mathbb{V}_{0}\right)$ эквивалентно следующему равенству:

$$
u(x, t)=\mathbb{A}^{-1}(v) .
$$

Из последнего уравнения следует, что $u(x, t) \in \mathbb{C}\left(\left[0, T_{0}\right) ; \mathbb{V}_{0}\right)$. Действительно,

$$
\left\|u(t)-u\left(t_{0}\right)\right\|_{0} \leqslant\left\|\mathbb{A}^{-1}(v)(t)-\mathbb{A}^{-1}(v)\left(t_{0}\right)\right\|_{0} \leqslant\left\|v(t)-v\left(t_{0}\right)\right\|_{0}^{*} \rightarrow+0, \quad t \rightarrow t_{0} .
$$

Значит, сушествует единственное решение $u$ уравнения $(4.5)$ класса $\mathbb{C}\left(\left[0, T_{0}\right) ; \mathbb{V}_{0}\right)$. Докажем теперь, что это решение принадлежит классу $\mathbb{C}^{1}\left(\left[0, T_{0}\right) ; \mathbb{V}_{0}\right)$.

Рассмотрим сначала производную Фреше оператора $\mathbb{A}$ :

$$
\mathbb{A}_{u}^{\prime}(u)=\mathbb{A}_{0}+\sum_{j=1}^{N} \mathbb{A}_{j, u}^{\prime}(u)
$$


В силу условий теоремы имеем

$$
\left\langle\mathbb{A}_{u}^{\prime}(u) u_{1}-\mathbb{A}_{u}^{\prime}(u) u_{2}, u_{1}-u_{2}\right\rangle \geqslant\left\|u_{1}-u_{2}\right\|_{0}^{2}
$$

для любых $u, u_{1}, u_{2} \in \mathbb{V}_{0}$. Значит, при любом фиксированном $u \in \mathbb{V}_{0}$ существует непрерывный по Липшицу обратный оператор $\left[\mathbb{A}_{u}^{\prime}\right]^{-1} \in \mathcal{L}\left(\mathbb{V}_{0}^{*} ; \mathbb{V}_{0}\right)$. Теперь рассмотрим следуюшее уравнение:

$$
\mathbb{A}_{0} u+\sum_{j=1}^{N} \mathbb{A}_{j}(u)=v(x, t) \in \mathbb{C}^{1}\left(\left[0, T_{0}\right) ; \mathbb{V}_{0}^{*}\right)
$$

Далее необходимо доказать обратимость оператора $\widehat{\mathbb{C}}=\mathbb{I}+\widehat{\mathbb{B}}$,

$$
\widehat{\mathbb{B}}=\sum_{j=1}^{N} \mathbb{A}_{0}^{-1} \mathbb{A}_{j, u}^{\prime}(u)
$$

где $\mathbb{A}_{j, u}^{\prime}(u)$ - производная Фреше при фиксированном элементе $u \in \mathbb{C}^{1}\left(\left[0, T_{0}\right) ; \mathbb{V}_{0}\right)$ оператора $\mathbb{A}_{j}$. Действительно, рассмотрим уравнение

$$
[\mathbb{I}+\widehat{\mathbb{B}}] w=0
$$

Докажем, что это уравнение имеет только тривиальное решение. С этой целью применим оператор $\mathbb{A}_{0}$ к обеим частям этого уравнения; тогда получим

$$
\mathbb{A}_{u}^{\prime} w=\mathbb{A}_{0} w+\sum_{j=1}^{N} \mathbb{A}_{j, u}^{\prime}(u) w=0 \quad \forall u \in \mathbb{V}_{0}
$$

Ранее мы доказали, что для оператора $\mathbb{A}_{u}^{\prime}$ определен непрерывный по Липшицу обратньй оператор $\left[\mathbb{A}_{u}^{\prime}\right]^{-1}$. Теперь применим этот оператор к обеим частям равенства (4.8). Все операции были эквивалентными, и поэтому мы приходим к выводу, что $w=0$.

Для функции $u \in \mathbb{C}^{1}\left(\left[0, T_{0}\right) ; \mathbb{V}_{0}\right)$ уравнение $(4.7)$ эквивалентно следующей задаче:

$$
\left[\mathbb{A}_{0}+\sum_{j=1}^{N} \mathbb{A}_{j, u}^{\prime}(u)\right] u^{\prime}=v^{\prime} \in \mathbb{C}\left(\left[0, T_{0}\right) ; \mathbb{V}_{0}^{*}\right), \quad u=\mathbb{A}^{-1} v
$$

Применим оператор $\mathbb{A}_{0}^{-1}$ к последнему уравнению; тогда получим

$$
[\mathbb{I}+\widehat{\mathbb{B}}] u^{\prime}=\mathbb{A}_{0}^{-1} v^{\prime}
$$

Введем оператор

$$
\widehat{\mathbb{C}}=\mathbb{I}+\widehat{\mathbb{B}}: \mathbb{V}_{0} \rightarrow \mathbb{V}_{0}^{*},
$$


для которого, как мы уже доказали, имеется обратный линейный ограниченный оператор. Поэтому (4.9) эквивалентно следующему равенству:

$$
u^{\prime}=\widehat{\mathbb{C}}^{-1} \mathbb{A}_{0}^{-1} v^{\prime} .
$$

Осталось доказать, что $u^{\prime}=\widehat{\mathbb{C}}^{-1} \mathbb{A}_{0}^{-1} v^{\prime} \in \mathbb{C}\left(\left[0, T_{0}\right) ; \mathbb{V}_{0}\right)$ при фиксированном $u \in \mathbb{C}\left(\left[0, T_{0}\right) ; \mathbb{V}_{0}\right)$. Действительно, справедлива следующая цепочка неравенств:

$$
\begin{aligned}
\left\|u^{\prime}(t)-u^{\prime}\left(t_{0}\right)\right\|_{0} \leqslant & \left\|\widehat{\mathbb{C}}^{-1}\left(t_{0}\right) \mathbb{A}_{0}^{-1}\left[v^{\prime}(t)-v^{\prime}\left(t_{0}\right)\right]\right\|_{0} \\
& +\left\|\left(\widehat{\mathbb{C}}^{-1}\left(t_{0}\right)-\widehat{\mathbb{C}}^{-1}(t)\right) \mathbb{A}_{0}^{-1} v^{\prime}\left(t_{0}\right)\right\|_{0} \\
\leqslant & C\left\|v^{\prime}(t)-v^{\prime}\left(t_{0}\right)\right\|_{0}^{*}+C\left\|\widehat{\mathbb{C}}^{-1}\left(t_{0}\right)-\widehat{\mathbb{C}}^{-1}(t)\right\|_{\mathbb{V}_{0} \rightarrow \mathbb{V}_{0}} .
\end{aligned}
$$

Отметим, что линейный при фиксированном $u \in \mathbb{C}\left(\left[0, T_{0}\right) ; \mathbb{V}_{0}\right)$ оператор $\widehat{\mathbb{C}}$ является непрерывным, а значит, в силу теоремы Банаха об обратном отображении оператор $\widehat{\mathbb{C}}^{-1}$ является линейным непрерывным, следовательно, в силу линейности - ограниченным. Стало быть, можно воспользоваться спектральным представлением для линейного ограниченного оператора $\widehat{\mathbb{C}}^{-1}: \mathbb{V}_{0} \rightarrow \mathbb{V}_{0}$.

Прежде всего введем резольвенту оператора $\widehat{\mathbb{C}}$ :

$$
\mathbb{R}(\lambda, \widehat{\mathbb{C}})=(\lambda \mathbb{I}-\widehat{\mathbb{C}})^{-1} .
$$

Пусть $\Gamma$ - окружность $|\lambda|=r$ с достаточно большим радиусом, бо́льшим, чем

$$
\sup _{t \in\left[t_{0}-\varepsilon, t_{0}+\varepsilon\right]}\|\widehat{\mathbb{C}}(t)\|_{\mathbb{V}_{0} \rightarrow \mathbb{V}_{0}} .
$$

Введенная величина определена корректно, поскольку при указанных $t \in\left[t_{0}-\varepsilon\right.$, $\left.t_{0}+\varepsilon\right] \subset\left[0, T_{0}\right)$ имеет место неравенство

$$
\sup _{t \in\left[t_{0}-\varepsilon, t_{0}+\varepsilon\right]}\|u(t)\|_{0}<+\infty .
$$

Теперь можно воспользоваться спектральньм представлением для операторов $\widehat{\mathbb{C}}^{-1}(t)$ и $\widehat{\mathbb{C}}^{-1}\left(t_{0}\right)$ с одним и тем же введенным ранее контуром $\Gamma$ :

$$
\widehat{\mathbb{C}}^{-1}(t)=\frac{1}{2 \pi i} \int_{\Gamma} \lambda^{-1} \mathbb{R}(\lambda, \widehat{\mathbb{C}}(t)) d \lambda, \quad \widehat{\mathbb{C}}^{-1}\left(t_{0}\right)=\frac{1}{2 \pi i} \int_{\Gamma} \lambda^{-1} \mathbb{R}\left(\lambda, \widehat{\mathbb{C}}\left(t_{0}\right)\right) d \lambda .
$$

Очевидно, имеем

$$
\widehat{\mathbb{C}}^{-1}(t)-\widehat{\mathbb{C}}^{-1}\left(t_{0}\right)=\frac{1}{2 \pi i} \int_{\Gamma} \lambda^{-1}\left[\mathbb{R}(\lambda, \widehat{\mathbb{C}}(t))-\mathbb{R}\left(\lambda, \widehat{\mathbb{C}}\left(t_{0}\right)\right)\right] d \lambda .
$$

Воспользуемся известным представлением для резольвент операторов:

$$
\mathbb{R}(\lambda, \widehat{\mathbb{C}}(t))-\mathbb{R}\left(\lambda, \widehat{\mathbb{C}}\left(t_{0}\right)\right)=\mathbb{R}\left(\lambda, \widehat{\mathbb{C}}\left(t_{0}\right)\right) \sum_{n=1}^{+\infty}\left[\left(\widehat{\mathbb{C}}(t)-\widehat{\mathbb{C}}\left(t_{0}\right)\right) \mathbb{R}\left(\lambda, \widehat{\mathbb{C}}\left(t_{0}\right)\right]^{n}\right.
$$


при условии

$$
\left\|\widehat{\mathbb{C}}\left(t_{0}\right)-\widehat{\mathbb{C}}(t)\right\|_{\mathbb{V}_{0} \rightarrow \mathbb{V}_{0}}\left\|\mathbb{R}\left(\lambda, \widehat{\mathbb{C}}\left(t_{0}\right)\right)\right\|_{\mathbb{V}_{0} \rightarrow \mathbb{V}_{0}} \leqslant \delta<1
$$

Справедливо следуюшее неравенство:

$$
\begin{aligned}
& \left\|\mathbb{R}(\lambda, \widehat{\mathbb{C}}(t))-\mathbb{R}\left(\lambda, \widehat{\mathbb{C}}\left(t_{0}\right)\right)\right\|_{\mathbb{V}_{0} \rightarrow \mathbb{V}_{0}} \\
& \quad \leqslant\left\|\mathbb{R}\left(\lambda, \widehat{\mathbb{C}}\left(t_{0}\right)\right)\right\|_{\mathbb{V}_{0} \rightarrow \mathbb{V}_{0}} \sum_{n=1}^{+\infty}\left\|\mathbb{R}\left(\lambda, \widehat{\mathbb{C}}\left(t_{0}\right)\right)\right\|_{\mathbb{V}_{0} \rightarrow \mathbb{V}_{0}}^{n}\left\|\widehat{\mathbb{C}}(t)-\widehat{\mathbb{C}}\left(t_{0}\right)\right\|_{\mathbb{V}_{0} \rightarrow \mathbb{V}_{0}}^{n} .
\end{aligned}
$$

Теперь заметим, что

$$
\widehat{\mathbb{C}}(t)-\widehat{\mathbb{C}}\left(t_{0}\right)=\mathbb{A}_{0}^{-1} \sum_{j=1}^{N}\left[\mathbb{A}_{j, u}^{\prime}(u(t))-\mathbb{A}_{j, u}^{\prime}\left(u\left(t_{0}\right)\right)\right]
$$

В силу непрерывности производных доказали, имеем $u \in \mathbb{C}\left(\left[0, T_{0}\right) ; \mathbb{V}_{0}\right)$. Следовательно,

$$
\begin{aligned}
& \left\|\widehat{\mathbb{C}}(t)-\widehat{\mathbb{C}}\left(t_{0}\right)\right\|_{\mathbb{V}_{0} \rightarrow \mathbb{V}_{0}} \leqslant \sum_{j=1}^{N}\left\|\mathbb{A}_{j, u}^{\prime}(u(t))-\mathbb{A}_{j, u}^{\prime}\left(u\left(t_{0}\right)\right)\right\|_{\mathbb{V}_{0} \rightarrow \mathbb{V}_{0}^{*}} \rightarrow+0, \\
& \left\|\mathbb{R}(\lambda, \widehat{\mathbb{C}}(t))-\mathbb{R}\left(\lambda, \widehat{\mathbb{C}}\left(t_{0}\right)\right)\right\|_{\mathbb{V}_{0} \rightarrow \mathbb{V}_{0}} \rightarrow+0, \\
& \left\|\widehat{\mathbb{C}}(t)^{-1}-\widehat{\mathbb{C}}\left(t_{0}\right)^{-1}\right\|_{\mathbb{V}_{0} \rightarrow \mathbb{V}_{0}} \rightarrow+0
\end{aligned}
$$

при $t \rightarrow t_{0}$. Значит, $u \in \mathbb{C}^{1}\left(\left[0, T_{0}\right) ; \mathbb{V}_{0}\right)$. Тем самым лемма доказана.

Шаг 2. Априорные оценки и разрушение решения. Справедлива следующая лемма.

ЛЕМма 4.2. Пусть

$$
\Phi(t) \equiv \frac{1}{2}\left\langle\mathbb{A}_{0} u, u\right\rangle_{0}+\sum_{j=1}^{N} \frac{p_{j}-1}{p_{j}}\left\langle\mathbb{A}_{j}(u), u\right\rangle_{j}, \quad \alpha \equiv \frac{q+2}{\bar{p}}
$$

Тогда для разных значений $\alpha=(q+2) / \bar{p}, \quad \bar{p}=\max _{j=\overline{1, n}} p_{j}$ справедливь следующие оценки:

1) если $\alpha \in(0,1)$, mо $T_{0}=+\infty, u$ тогда

$$
\Phi(t) \leqslant\left[\Phi_{0}^{1-\alpha}+(1-\alpha) \bar{A} t\right]^{1 /(1-\alpha)}
$$

2) если $\alpha=1$, mо $T_{0}=+\infty$, и тогда $\Phi(t) \leqslant \Phi_{0} \exp \{\bar{A} t\}$;

3) если $\alpha>1$ и вьполнень условия

$$
\Phi^{\prime}(0)>\frac{\beta}{\alpha-1} \Phi(0), \quad \Phi^{\prime}(0)>\sqrt{\frac{\gamma}{\alpha-1}} \Phi(0),
$$


mо найдется такое $T_{0} \in\left[T_{1}, T_{2}\right]$, что $\lim _{t \uparrow T_{0}} \Phi(t)=+\infty ;$ здесь

$$
\begin{gathered}
\Phi(t) \equiv \frac{1}{2}\left\langle\mathbb{A}_{0} u, u\right\rangle_{0}+\sum_{j=1}^{N} \frac{p_{j}-1}{p_{j}}\left\langle\mathbb{A}_{j}(u), u\right\rangle_{j}, \quad \Phi_{0} \equiv \Phi(0), \\
T_{1}=\frac{2}{q} \Phi_{0}^{-q / 2} \bar{B}^{-1}, \quad T_{2}=\Phi_{0}^{1-\alpha} A^{-1}, \quad \bar{B}=2^{(q+2) / 2} B C_{1}^{q+2}, \\
\bar{A} \equiv C_{j^{*}}^{q+2} M\left(\frac{\bar{p}}{\bar{p}-1}\right)^{(q+2) / \bar{p}}, \quad|v|_{0} \leqslant C_{j^{*}}\left\langle\mathbb{A}_{j^{*}} v, v\right\rangle_{j^{*}}^{1 / p_{j}}, \\
\Phi_{0} \equiv \Phi(0), \quad \Phi^{\prime}(0) \equiv\left(\mathbb{F}\left(u_{0}\right), u_{0}\right)_{0}-\left(\mathbb{L}_{0}, u_{0}\right)_{1}, \\
\frac{\bar{p}+q+2}{2 \bar{p}}, \quad \beta \equiv \frac{(q+2)(q+1)}{q+2-\bar{p}}, \quad \gamma \equiv \frac{4(q+1)^{2}}{q+2-\bar{p}}, \quad q+2>\bar{p} \equiv \max _{j=\overline{1, n}} p_{j}, \\
A^{2} \equiv(\alpha-1)^{2} \Phi_{0}^{-2 \alpha}\left[\left(\Phi^{\prime}(0)\right)^{2}-\frac{\gamma}{\alpha-1} \Phi^{2}(0)\right],
\end{gathered}
$$

$C_{1}$ - константа наилучшего вложения $\mathbb{V} \hookrightarrow \mathbb{W}_{0}, \quad B$ - константа из условия $\mathbb{F}, 4)$.

ДокАЗАТЕЛьСтво. Пусть $u \in \mathbb{C}^{1}\left(\left[0, T_{0}\right) ; \mathbb{V}_{0}\right)$ - максимальное решение задачи (1.1). Рассмотрим в равенстве (4.1) функцию $v=u(t)$; после интегрирования по частям получим следуюшее равенство:

$$
\frac{d}{d t}\left[\frac{1}{2}\left\langle\mathbb{A}_{0} u, u\right\rangle_{0}+\sum_{j=1}^{N} \frac{p_{j}-1}{p_{j}}\left\langle\mathbb{A}_{j}(u), u\right\rangle_{j}\right]=(\mathbb{F}(u), u)_{0}-(\mathbb{L} u, u)_{1}
$$

Теперь пусть в (4.1) $v=u^{\prime}(t)$; интегрируя по частям, имеем

$$
\left\langle\mathbb{A}_{0} u^{\prime}, u^{\prime}\right\rangle_{0}+\sum_{j=1}^{N}\left\langle\left(\mathbb{A}_{j}(u)\right)^{\prime}, u^{\prime}\right\rangle_{j}=\frac{1}{q+2} \frac{d}{d t}(\mathbb{F}(u), u)_{0}-\frac{1}{2} \frac{d}{d t}(\mathbb{L} u, u)_{1}-\left\langle\mathbb{D P}(u), u^{\prime}\right\rangle_{0}
$$

Интегрируя уравнение (4.12) по $t \in(0, T)$, получим

$$
\Phi(t)=\Phi_{0}+\int_{0}^{t}\left[(\mathbb{F}(u), u)_{0}-(\mathbb{L} u, u)_{1}\right] d s
$$

где

$$
\Phi(t) \equiv \frac{1}{2}\left\langle\mathbb{A}_{0} u, u\right\rangle_{0}+\sum_{j=1}^{N} \frac{p_{j}-1}{p_{j}}\left\langle\mathbb{A}_{j}(u), u\right\rangle_{j}, \quad \Phi_{0}=\Phi(0)
$$

Поскольку по условию $\mathbb{V}_{0} \subset \mathbb{W}_{0}$, то найдется постоянная $C_{1}>0$ такая, что

$$
|v|_{0} \leqslant C_{1}\left\langle\mathbb{A}_{0} v, v\right\rangle_{0}^{1 / 2}
$$


и из (4.14) вытекает неравенство

$$
\begin{aligned}
\Phi(t) & \leqslant \Phi_{0}+M C_{1}^{q+2} \int_{0}^{t}\left\langle\mathbb{A}_{0} v, v\right\rangle_{0}^{(q+2) / 2} d s \\
& \leqslant \Phi_{0}+\bar{B} \int_{0}^{t} \Phi^{(q+2) / 2}(s) d s, \quad \bar{B} \equiv 2^{(q+2) / 2} M C_{1}^{q+2} .
\end{aligned}
$$

По теореме Бихари [29] получаем

$$
\Phi(t) \leqslant \frac{\Phi_{0}}{\left[1-\frac{q}{2} \Phi_{0}^{q / 2} \bar{B} t\right]^{2 / q}} \quad \forall t \in\left[0, T_{1}\right), \quad T_{1} \equiv \frac{2}{q} \Phi_{0}^{-q / 2} \bar{B}^{-1} .
$$

Стало быть, в случае $\bar{p}<q+2$ имеем $T_{0} \geqslant T_{1}$.

Пусть теперь $\bar{p} \geqslant q+2$. Воспользуемся тем, что в данном случае найдется $j^{*} \in \overline{1, N}$, при котором $p_{j^{*}}=\bar{p}$ и $p_{j^{*}} \geqslant q+2$, такое, что $\mathbb{V}_{j^{*}} \subset \mathbb{W}_{0}$. Пусть $C_{j^{*}-}$ константа вложения $\mathbb{V}_{j *} \subset \mathbb{W}_{0}$ :

$$
\begin{gathered}
|v|_{0} \leqslant C_{j^{*}}\left\langle\mathbb{A}_{j^{*}}(v), v\right\rangle_{j^{*}}^{1 / p_{j^{*}}} \\
\Phi(t) \leqslant \Phi_{0}+C_{j^{*}}^{q+2} M \int_{0}^{t}\left\langle\mathbb{A}_{j^{*}}(v), v\right\rangle_{j^{*}}^{(q+2) / p_{j^{*}}} d s \\
\leqslant \Phi_{0}+C_{j^{*}}^{q+2} M\left(\frac{\bar{p}}{\bar{p}-1}\right)^{(q+2) / \bar{p}} \int_{0}^{t} \Phi^{(q+2) / \bar{p}}(s) d s .
\end{gathered}
$$

Рассмотрим два случая: $\alpha=1, \alpha \in(0,1)$, где $\alpha \equiv \frac{q+2}{\bar{p}}$.

В первом случае по теореме Гронуолла-Беллмана [29] имеем

$$
\Phi(t) \leqslant \Phi_{0} \exp \{\bar{A} t\}, \quad \bar{A} \equiv C_{j^{*}}^{q+2} M\left(\frac{\bar{p}}{\bar{p}-1}\right)^{(q+2) / \bar{p}} .
$$

Во втором случае, когда $\alpha \in(0,1)$, по теореме Бихари получим

$$
\Phi(t) \leqslant\left[\Phi_{0}^{1-\alpha}+(1-\alpha) \bar{A} t\right]^{1 /(1-\alpha)} .
$$

Из соотношений (4.16) и (4.17) следует, что $T_{0}=+\infty$ в случае $\alpha \in(0,1]$.

Теперь из соотношений (4.12) и (4.13) точно так же, как и на шаге 5 доказательства теоремы 3.1, выводим следуюшее обыкновенное дифференциальное неравенство второго порядка:

$$
\Phi^{\prime \prime} \Phi-\alpha\left(\Phi^{\prime}\right)^{2}+\beta \Phi^{\prime} \Phi+\gamma \Phi^{2} \geqslant 0
$$

где

$$
\alpha \equiv \frac{\bar{p}+q+2}{2 \bar{p}}, \quad \beta \equiv \frac{(q+2)(q+1)}{q+2-\bar{p}}, \quad \gamma \equiv \frac{4(q+1)^{2}}{q+2-\bar{p}}, \quad q+2>\bar{p} \equiv \max _{j=\overline{1, n}} p_{j} .
$$

Интегрируя дифференщиальное неравенство (4.18), с учетом (4.12) и (4.13) и неравенств

получим

$$
\Phi^{\prime}(0)>\frac{\beta}{\alpha-1} \Phi_{0}, \quad \Phi^{\prime}(0)>\sqrt{\frac{\gamma}{\alpha-1}} \Phi(0)
$$

$$
\Phi \geqslant \frac{e^{\theta t} A^{1 /\left(1-\alpha_{1}\right)}}{\left[T_{2}-t\right]^{1 /\left(\alpha_{1}-1\right)}} \quad \forall t \in\left[0, T_{2}\right), \quad T_{2} \equiv \Phi_{0}^{1-\alpha_{1}} A^{-1}, \quad \theta=\frac{\beta}{\alpha-1} .
$$

Таким образом, теорема 4.1 доказана. 


\section{§5. Примеры}

В примерах используются стандартные теоремы вложения Соболева, которые можно найти, например, в [32].

Во всех примерах $\mathbb{H}=\mathbb{L}^{2}(\Omega)$.

ПримеР 5.1. Рассмотрим следуюшую задачу:

$$
\begin{gathered}
\frac{\partial}{\partial t}\left(\Delta u-u+\sum_{j=1}^{N} \operatorname{div}\left(|\nabla u|^{p_{j}-2} \nabla u\right)\right)-u+u \frac{\partial u}{\partial x_{1}}+u^{3}=0, \\
\left.u\right|_{\partial \Omega}=0, \quad u(x, 0)=u_{0}(x),
\end{gathered}
$$

где $\Omega \subset \mathbb{R}^{N}$ - ограниченная область с гладкой границей класса $\mathbb{C}^{(2, \delta)}, \delta \in(0,1]$, а операторные коэффициенты имеют вид

$$
\begin{gathered}
\mathbb{A}_{0} u \equiv-\Delta u+u: \mathbb{H}_{0}^{1}(\Omega) \rightarrow \mathbb{H}^{-1}(\Omega), \\
\mathbb{A}_{j}(u) \equiv-\operatorname{div}\left(|\nabla u|^{p_{j}-2} \nabla u\right): \mathbb{W}_{0}^{1, p_{j}}(\Omega) \rightarrow \mathbb{W}^{-1, p_{j}^{\prime}}(\Omega), \quad p_{j}>2, \\
\mathbb{F}(u) \equiv u^{3}: \mathbb{L}^{4}(\Omega) \rightarrow \mathbb{L}^{4 / 3}(\Omega), \quad \mathbb{P}(u) \equiv u^{2}: \mathbb{L}^{4}(\Omega) \rightarrow \mathbb{L}^{2}(\Omega), \\
\mathbb{D} v \equiv \frac{\partial v}{\partial x_{1}}: \mathbb{L}^{2}(\Omega) \rightarrow \mathbb{H}^{-1}(\Omega), \\
\mathbb{L} u=\mathbb{I} u: \mathbb{W}_{1}=\mathbb{L}^{2}(\Omega) \rightarrow \mathbb{L}^{2}(\Omega)=\mathbb{W}_{1}^{*}, \\
\mathbb{V}_{0} \equiv \mathbb{H}_{0}^{1}(\Omega), \quad \mathbb{V}_{j} \equiv \mathbb{W}_{0}^{1, p_{j}}(\Omega), \\
\mathbb{W}_{0} \equiv \mathbb{L}^{4}(\Omega), \quad \mathbb{W}_{2} \equiv \mathbb{L}^{4}(\Omega), \quad \mathbb{W}_{3} \equiv \mathbb{L}^{2}(\Omega), \quad \mathbb{W}_{4} \equiv \mathbb{H}^{-1}(\Omega), \\
\mathbb{V}_{0}^{*} \equiv \mathbb{H}^{-1}(\Omega), \quad \mathbb{V}_{j}^{*} \equiv \mathbb{W}^{-1, p_{j}^{\prime}}(\Omega), \quad \mathbb{W}_{0}^{*} \equiv \mathbb{L}^{4 / 3}(\Omega), \\
\mathbb{V} \equiv \mathbb{H}_{0}^{1}(\Omega) \cap\left(\bigcap_{j=1}^{N} \mathbb{W}_{0}^{1, p_{j}}(\Omega)\right) .
\end{gathered}
$$

Вложение $\mathbb{V}_{0} \equiv \mathbb{H}_{0}^{1}(\Omega) \subset \mathbb{W}_{0} \equiv \mathbb{L}^{4}(\Omega)$ имеет место при условии $N \leqslant 4$.

Наконец вложение $\mathbb{V} \hookrightarrow \mathbb{W}_{0}=\mathbb{W}_{2}$ справедливо при условиях

$$
\bigcap_{j=1}^{N} \mathbb{W}_{0}^{1, p_{j}}(\Omega) \hookrightarrow \mathbb{L}^{q+2}(\Omega),
$$

что, в свою очередь, справедливо при условиях

$$
N \leqslant \bar{p} \equiv \max _{j=\overline{1, n}} p_{j}, \quad 4<\frac{N \bar{p}}{N-\bar{p}} \text { при } \quad N>\bar{p} .
$$

Предположим, что выполнены следуюшие условия, налагаемые на начальную функцию $u_{0} \in \mathbb{V}$ :

$$
\Phi^{\prime}(0)>\frac{\beta}{\alpha-1} \Phi(0), \quad \Phi^{\prime}(0)>\sqrt{\frac{\gamma}{\alpha-1}} \Phi(0),
$$


где

$$
\begin{array}{cl}
\Phi(0)=\frac{1}{2}\left\|\nabla u_{0}\right\|_{2}^{2}+\sum_{j=1}^{N} \frac{p_{j}-1}{p_{j}}\|\nabla u\|_{p_{j}}^{p_{j}}, \quad \Phi^{\prime}(0)=\left\|u_{0}\right\|_{4}^{4}-\left\|u_{0}\right\|_{2}^{2}, \\
\alpha=\frac{\bar{p}+4}{2 \bar{p}}, \quad \beta=\frac{12}{4-\bar{p}}, \quad \gamma=\frac{36}{4-\bar{p}}
\end{array}
$$

при $\bar{p}<4$.

Можно проверить, что операторные коэффициенты удовлетворяют всем условиям теоремы 3.1 .

ПримеР 5.2. Рассмотрим следуюшую задачу:

$$
\begin{gathered}
\frac{\partial}{\partial t}\left(-\Delta^{2} u+\Delta u+\operatorname{div}\left(|\nabla u|^{p-2} \nabla u\right)\right)+\Delta u+u \frac{\partial u}{\partial x_{1}}-\operatorname{div}\left(|\nabla u|^{2} \nabla u\right)=0, \\
\left.u\right|_{\partial \Omega}=\left.\frac{\partial u}{\partial \mathbf{n}}\right|_{\partial \Omega}=0, \quad u(0, x)=u_{0}(x),
\end{gathered}
$$

где $\Omega \subset \mathbb{R}^{N}$ - ограниченная область с гладкой гранищей $\partial \Omega$ класса $\mathbb{C}^{(4, \delta)}, \delta \in$ $(0,1]$, а операторные коэффициенты имеют вид

$$
\begin{gathered}
\mathbb{A}_{0} u \equiv \Delta^{2} u-\Delta u: \mathbb{H}_{0}^{2}(\Omega) \rightarrow \mathbb{H}^{-2}(\Omega), \\
\mathbb{F}(u) \equiv-\operatorname{div}\left(|\nabla u|^{2} \nabla u\right): \mathbb{W}_{0}^{1,4}(\Omega) \rightarrow \mathbb{W}^{-1,4 / 3}(\Omega), \\
\mathbb{A}_{1}(u) \equiv-\operatorname{div}\left(|\nabla u|^{p-2} \nabla u\right): \mathbb{W}_{0}^{1, p}(\Omega) \rightarrow \mathbb{W}^{-1, p^{\prime}}(\Omega), \\
\mathbb{P}(u)=u^{2}: \mathbb{H}_{0}^{1}(\Omega) \subset \mathbb{L}^{4}(\Omega) \rightarrow \mathbb{L}^{2}(\Omega), \\
\mathbb{D} v=\frac{\partial v}{\partial x_{1}}: \mathbb{L}^{2}(\Omega) \rightarrow \mathbb{H}^{-1}(\Omega), \quad \mathbb{L} u=-\Delta u: \mathbb{W}_{1}=\mathbb{H}_{0}^{1}(\Omega) \rightarrow \mathbb{H}^{-1}(\Omega)=\mathbb{W}_{1}^{*}, \\
\mathbb{V}_{0} \equiv \mathbb{H}_{0}^{2}(\Omega), \quad \mathbb{W}_{0} \equiv \mathbb{W}_{0}^{1,4}(\Omega), \quad \mathbb{W}_{2} \equiv \mathbb{L}^{4}(\Omega), \\
\mathbb{V}_{1} \equiv \mathbb{W}_{0}^{1, p}(\Omega), \quad \mathbb{W}_{3} \equiv \mathbb{L}^{2}(\Omega), \quad \mathbb{W}_{4} \equiv \mathbb{H}^{-1}(\Omega), \\
\mathbb{V}_{0}^{*} \equiv \mathbb{H}^{-2}(\Omega), \quad \mathbb{W}_{0}^{*} \equiv \mathbb{W}^{-1,4 / 3}(\Omega), \quad \mathbb{V}_{1}^{*} \equiv \mathbb{W}^{-1, p^{\prime}}(\Omega), \quad p^{\prime}=\frac{p}{p-1}, \\
\mathbb{V} \equiv \mathbb{H}_{0}^{2}(\Omega) \cap \mathbb{W}_{0}^{1, p}(\Omega) .
\end{gathered}
$$

Вложение $\mathbb{V} \hookrightarrow \mathbb{W}_{0} \subset \mathbb{W}_{1}$ имеет место при $N \leqslant 3$ и $p \in(2,6]$. Также имеют место вложения $\mathbb{V}_{0} \hookrightarrow \mathbb{W}_{1}, \mathbb{V}_{0} \subseteq \mathbb{W}_{0} \subseteq \mathbb{W}_{2}$.

Предположим теперь, что выполнены следуюшие условия, налагаемые на начальную функцию $u_{0} \in \mathbb{H}_{0}^{2}(\Omega)$ :

где

$$
\Phi^{\prime}(0)>\frac{\beta}{\alpha-1} \Phi(0), \quad \Phi^{\prime}(0)>\sqrt{\frac{\gamma}{\alpha-1}} \Phi(0),
$$

$$
\begin{gathered}
\alpha=\frac{4+p}{2 p}, \quad \beta=\frac{12}{4-p}, \quad \gamma=\frac{36}{4-p}, \\
\Phi(0)=\frac{1}{2}\left\|\Delta u_{0}\right\|_{2}^{2}+\frac{1}{2}\left\|\nabla u_{0}\right\|_{2}^{2}+\frac{p-1}{p}\left\|\nabla u_{0}\right\|_{p}^{p}, \\
\Phi^{\prime}(0)=\left\|\nabla u_{0}\right\|_{4}^{4}-\left\|\nabla u_{0}\right\|_{2}^{2}
\end{gathered}
$$

при $p<4$. Таким образом, выполнены все условия теоремы 3.1 . 
ПримеР 5.3. Рассмотрим следующую задачу:

$$
\begin{gathered}
\frac{\partial}{\partial t}\left(-\Delta^{2} u+\Delta u+\operatorname{div}\left(|\nabla u|^{p-2} \nabla u\right)\right)+\Delta u-\operatorname{div}\left(|\nabla u|^{2} \nabla u\right) \\
+\beta_{1} \frac{\partial}{\partial x_{1}}\left(\frac{\partial u}{\partial x_{2}} \frac{\partial u}{\partial x_{3}}\right)+\beta_{2} \frac{\partial}{\partial x_{2}}\left(\frac{\partial u}{\partial x_{3}} \frac{\partial u}{\partial x_{1}}\right)+\beta_{3} \frac{\partial}{\partial x_{3}}\left(\frac{\partial u}{\partial x_{1}} \frac{\partial u}{\partial x_{2}}\right)=0 \\
\left.u\right|_{\partial \Omega}=\left.\frac{\partial u}{\partial \mathbf{n}}\right|_{\partial \Omega}=0, \quad u(x, 0)=u_{0}(x),
\end{gathered}
$$

где $\beta_{1}+\beta_{2}+\beta_{3}=0,\left|\beta_{1}\right|+\left|\beta_{2}\right|+\left|\beta_{3}\right|>0, \Omega \subset \mathbb{R}^{N}$ - ограниченная область с гладкой границей $\partial \Omega$ класса $\mathbb{C}^{(4, \delta)}, \delta \in(0,1]$, а операторные коэффициенты имеют вид

$$
\begin{gathered}
\mathbb{A}_{1} u=-\operatorname{div}\left(|\nabla u|^{p-2} \nabla u\right): \mathbb{V}_{1}=\mathbb{W}_{0}^{1, p}(\Omega) \rightarrow \mathbb{V}_{1}^{*}=\mathbb{W}^{-1, p^{\prime}}(\Omega), \\
\mathbb{L} u=-\Delta u: \mathbb{W}_{1}=\mathbb{H}_{0}^{1}(\Omega) \rightarrow \mathbb{W}_{1}^{*}=\mathbb{H}^{-1}(\Omega), \\
\mathbb{A}_{0} u \equiv \Delta^{2} u-\Delta u: \mathbb{H}_{0}^{2}(\Omega) \rightarrow \mathbb{H}^{-2}(\Omega), \\
\mathbb{F}(u) \equiv-\operatorname{div}\left(|\nabla u|^{2} \nabla u\right): \mathbb{W}_{0}^{1,4}(\Omega) \rightarrow \mathbb{W}^{-1,4 / 3}(\Omega), \\
\mathbb{P}(u)=\beta_{1} \frac{\partial u}{\partial x_{2}} \frac{\partial u}{\partial x_{3}} \mathbf{e}_{1}+\beta_{2} \frac{\partial u}{\partial x_{3}} \frac{\partial u}{\partial x_{1}} \mathbf{e}_{2} \\
+\beta_{3} \frac{\partial u}{\partial x_{1}} \frac{\partial u}{\partial x_{2}} \mathbf{e}_{3}: \mathbb{W}_{0}^{1,4}(\Omega) \rightarrow \mathbb{L}^{2}(\Omega) \times \mathbb{L}^{2}(\Omega) \times \mathbb{L}^{2}(\Omega), \\
\mathbb{D} \mathbf{v} \equiv \operatorname{div}(\mathbf{v}): \mathbb{L}^{2}(\Omega) \times \mathbb{L}^{2}(\Omega) \times \mathbb{L}^{2}(\Omega) \rightarrow \mathbb{H}^{-1}(\Omega), \\
\mathbb{V}_{0} \equiv \mathbb{H}_{0}^{2}(\Omega), \quad \mathbb{W}_{0} \equiv \mathbb{W}_{0}^{1,4}(\Omega), \\
\mathbb{W}_{2} \equiv \mathbb{W}_{0}^{1,4}(\Omega), \quad \mathbb{W}_{3} \equiv \mathbb{L}^{2}(\Omega) \times \mathbb{L}^{2}(\Omega) \times \mathbb{L}^{2}(\Omega), \\
\mathbb{W}_{4} \equiv \mathbb{H}^{-1}(\Omega), \quad \mathbb{V}_{0}^{*} \equiv \mathbb{H}^{-2}(\Omega), \quad \mathbb{W}_{0}^{*} \equiv \mathbb{W}^{-1,4 / 3}(\Omega), \\
\mathbb{V} \equiv \mathbb{H}_{0}^{2}(\Omega)
\end{gathered}
$$

при $N \leqslant 3$ и $p \in(2,6]$. Кроме того, имеют место вложения $\mathbb{V} \hookrightarrow \mathbb{W}_{0} \subset \mathbb{W}_{1}$, $\mathbb{V}_{0} \subseteq \mathbb{W}_{1}, \mathbb{V}_{0} \subseteq \mathbb{W}_{0} \subseteq \mathbb{W}_{2}$

Предположим, что выполнены следующие условия, налагаемые на начальную функцию $u_{0} \in \mathbb{H}_{0}^{2}(\Omega)$ :

$$
\Phi^{\prime}(0)>\frac{\beta}{\alpha-1} \Phi(0), \quad \Phi^{\prime}(0)>\sqrt{\frac{\gamma}{\alpha-1}} \Phi(0),
$$

где

$$
\begin{gathered}
\alpha=\frac{4+p}{2 p}, \quad \beta=\frac{12}{4-p}, \quad \gamma=\frac{36}{4-p}, \\
\Phi(0)=\frac{1}{2}\left\|\Delta u_{0}\right\|_{2}^{2}+\frac{1}{2}\left\|\nabla u_{0}\right\|_{2}^{2}+\frac{p-1}{p}\left\|\nabla u_{0}\right\|_{p}^{p}, \\
\Phi^{\prime}(0)=\left\|\nabla u_{0}\right\|_{4}^{4}-\left\|\nabla u_{0}\right\|_{2}^{2}
\end{gathered}
$$

при $p<4$. Таким образом, выполнены все условия теоремы 3.1 . 
ПримеР 5.4. Рассмотрим следующую задачу:

$$
\begin{gathered}
\frac{\partial}{\partial t}\left(\Delta u-u-|u|^{p_{1}-2} u\right)+\Delta u+\frac{\partial|u|^{q_{2}+1}}{\partial x_{1}}+|u|^{2 q_{2}} u=0, \\
\left.u\right|_{\partial \Omega}=0, \quad u(x, 0)=u_{0}(x),
\end{gathered}
$$

где $\Omega \subset \mathbb{R}^{N}$ - ограниченная область с гладкой гранищей $\partial \Omega$ класса $\mathbb{C}^{(2, \delta)}, \delta \in$ $(0,1]$, а операторные коэффициенты имеют вид

$$
\begin{gathered}
\mathbb{A}_{0} u \equiv-\Delta u+u: \mathbb{H}_{0}^{1}(\Omega) \rightarrow \mathbb{H}^{-1}(\Omega), \quad \mathbb{A}_{1}(u) \equiv|u|^{p_{1}-2} u: \mathbb{L}^{p_{1}}(\Omega) \rightarrow \mathbb{L}^{p_{1}^{\prime}}(\Omega), \\
\mathbb{F}(u) \equiv|u|^{q_{2}} u: \mathbb{L}^{q_{2}+2}(\Omega) \rightarrow \mathbb{L}^{q_{2}^{\prime}}(\Omega), \quad q_{2}^{\prime}=\frac{q_{2}+2}{q_{2}+1}, \\
\mathbb{P}(u) \equiv|u|^{q_{2}+1}: \mathbb{L}_{0}^{2 q_{2}+2}(\Omega) \rightarrow \mathbb{L}^{2}(\Omega), \quad \mathbb{D} v \equiv \frac{\partial v}{\partial x_{1}}: \mathbb{L}^{2}(\Omega) \rightarrow \mathbb{H}^{-1}(\Omega), \\
\mathbb{L} u \equiv-\Delta u: \mathbb{W}_{1}=\mathbb{H}_{0}^{1}(\Omega) \rightarrow \mathbb{W}_{1}^{*}=\mathbb{H}^{-1}(\Omega), \\
\mathbb{V}_{0} \equiv \mathbb{H}_{0}^{1}(\Omega), \quad \mathbb{V}_{1} \equiv \mathbb{L}^{p_{1}}(\Omega), \quad \mathbb{W}_{0} \equiv \mathbb{L}^{2 q_{2}+2}(\Omega), \\
\mathbb{W}_{2} \equiv \mathbb{L}^{2 q_{2}+2}(\Omega), \quad \mathbb{W}_{3} \equiv \mathbb{L}^{2}(\Omega), \quad \mathbb{W}_{4} \equiv \mathbb{H}^{-1}(\Omega), \\
\mathbb{V}_{0}^{*} \equiv \mathbb{H}^{-1}(\Omega), \quad \mathbb{V}_{1}^{*} \equiv \mathbb{L}^{p_{1}^{\prime}}(\Omega), \quad \mathbb{W}_{0}^{*} \equiv \mathbb{L}^{q_{2}^{\prime}}(\Omega), \\
p_{1}=q_{1}+2, \quad q_{1}>0, \quad p_{1}^{\prime} \equiv \frac{p_{1}}{p_{1}-1}, \quad q_{2}^{\prime} \equiv \frac{q_{2}+2}{q_{2}+1}, \quad q_{2}>0 .
\end{gathered}
$$

Вложение $\mathbb{V}_{0} \subset \mathbb{W}_{0}$ имеет место либо при условии $0<q_{2} \leqslant 2 /(N-2), N \geqslant 3$, либо при условии $0<q_{2}<+\infty, N=1,2$. Это означает, что

$$
\mathbb{V} \equiv \mathbb{H}_{0}^{1}(\Omega) \cap \mathbb{L}^{q_{1}+2}(\Omega) \hookrightarrow \mathbb{W}_{0} \equiv \mathbb{L}^{q+2}(\Omega) .
$$

Кроме того, $\mathbb{W}_{0}=\mathbb{W}_{2}$. Предположим, что

$$
\Phi^{\prime}(0)>\frac{\beta}{\alpha-1} \Phi(0), \quad \Phi^{\prime}(0)>\sqrt{\frac{\gamma}{\alpha-1}} \Phi(0),
$$

где

$$
\begin{aligned}
\Phi^{\prime}(0) & =\left\|u_{0}\right\|_{2 q_{2}+2}^{2 q_{2}+2}-\left\|\nabla u_{0}\right\|_{2}^{2}, \quad \Phi(0)=\frac{1}{2}\left\|\nabla u_{0}\right\|_{2}^{2}+\frac{1}{2}\left\|u_{0}\right\|_{2}^{2}+\frac{1}{p_{1}}\left\|u_{0}\right\|_{p_{1}}^{p_{1}}, \\
\alpha & =\frac{2 q_{2}+2+p_{1}}{2 p_{1}}, \quad \beta=\frac{2\left(q_{2}+1\right)\left(2 q_{2}+1\right)}{2 q_{2}+2-p_{1}}, \quad \gamma=\frac{4\left(2 q_{2}+1\right)^{2}}{2 q_{2}+2-p_{1}}
\end{aligned}
$$

при условии $\bar{p}=p_{1}<2 q_{2}+2$.

Проверим монотонность производной Фреше оператора $\mathbb{A}_{1}(u)=|u|^{q_{1}} u$ в указанном в формулировке теоремы 4.1 смысле. Действительно, производная Фреше имеет вид

$$
\begin{gathered}
\mathbb{A}_{1, u}^{\prime}(u) h \equiv\left(q_{1}+1\right)|u|^{q_{1}} h: \mathbb{L}^{q_{1}+2}(\Omega) \rightarrow \mathbb{L}^{q_{1}^{\prime}}(\Omega), \quad q_{1}^{\prime} \equiv \frac{q_{1}+2}{q_{1}+1}, \quad \forall h \in \mathbb{L}^{q_{1}+2}(\Omega), \\
\left\langle\mathbb{A}_{1, u}^{\prime}(u) u_{1}-\mathbb{A}_{1, u}^{\prime}(u) u_{2}, u_{1}-u_{2}\right\rangle_{1}=\left(q_{1}+1\right)\left\langle|u|^{q_{1}} u_{1}-|u|^{q_{1}} u_{2}, u_{1}-u_{2}\right\rangle_{1} \\
=\left(q_{1}+1\right) \int_{\Omega}\left(|u|^{q_{1}} u_{1}-|u|^{q_{1}} u_{2}\right)\left(u_{1}-u_{2}\right) d x \geqslant 0 \quad \forall u, u_{1}, u_{2} \in \mathbb{L}^{q_{1}+2}(\Omega),
\end{gathered}
$$


где $q_{1}=p_{1}-2$. Докажем теперь, что при условии $\left\|\nabla u_{n}-\nabla u\right\|_{2} \rightarrow 0$, когда $n \rightarrow+\infty$, имеет место сходимость производных Фреше в равномерной операторной топологии $\mathcal{L}\left(\mathbb{H}_{0}^{1}(\Omega), \mathbb{H}^{-1}(\Omega)\right)$. Действительно,

$$
\begin{aligned}
& \left\|\mathbb{A}_{1, u}^{\prime}(u)-\mathbb{A}_{1, u}^{\prime}\left(u_{n}\right)\right\|_{\mathbb{H}_{0}^{1} \rightarrow \mathbb{H}^{-1}}=\sup _{\|\nabla h\|_{2}=1}\left\|\mathbb{A}_{1, u}^{\prime}(u) h-\mathbb{A}_{1, u}^{\prime}\left(u_{n}\right) h\right\|_{-1} \\
& \leqslant \sup _{\|\nabla h\|_{2}=1}\left\|\mathbb{A}_{1, u}^{\prime}(u) h-\mathbb{A}_{1, u}^{\prime}\left(u_{n}\right) h\right\|_{(q+2) /(q+1)} \\
& \leqslant(q+1) \sup _{\|\nabla h\|_{2}=1}\left(\int_{\Omega}\left|\left[|u|^{q}-\left|u_{n}\right|^{q}\right] h\right|^{(q+2) /(q+1)} d x\right)^{(q+1) /(q+2)} .
\end{aligned}
$$

Рассмотрим отдельно интеграл

$$
\begin{aligned}
J_{n} & =\int_{\Omega}\left|\left[|u|^{q}-\left|u_{n}\right|^{q}\right] h\right|^{(q+2) /(q+1)} d x \\
& \leqslant\left.\int_{\Omega}|| u\right|^{q}-\left.\left|u_{n}\right|^{q}\right|^{(q+2) /(q+1)}|h|^{(q+2) /(q+1)} d x \\
& \leqslant\left(\left.\int_{\Omega}|| u\right|^{q}-\left.\left|u_{n}\right|^{q}\right|^{(q+2) / q} d x\right)^{q /(q+1)}\left(\int_{\Omega}|h|^{q+2} d x\right)^{1 /(q+1)} \\
& \leqslant\|\nabla h\|_{2}^{(q+2) /(q+1)}\left(\left.\int_{\Omega}|| u\right|^{q}-\left.\left|u_{n}\right|^{q}\right|^{(q+2) / q} d x\right)^{q /(q+1)} \\
& \leqslant C\left(\left.\int_{\Omega}|| u\right|^{q}-\left.\left|u_{n}\right|^{q}\right|^{(q+2) / q} d x\right)^{q /(q+1)} \\
& \leqslant C \max \left\{\|\nabla u\|_{2}^{(q+2) q /(q+1)},\left\|\nabla u_{n}\right\|_{2}^{(q+2) q /(q+1)}\right\} \leqslant C .
\end{aligned}
$$

Отметим, что поскольку $\left\|\nabla u-\nabla u_{n}\right\|_{2} \rightarrow+0$ при $n \rightarrow+\infty$, то $u_{n} \rightarrow u$ сильно в $\mathbb{L}^{q+2}(\Omega)$. Рассмотрим оператор $f(x, u)=|u|^{q}: \mathbb{L}^{q+1}(\Omega) \rightarrow \mathbb{L}^{(q+1) / q}$. Значит, по теореме Вайнберга-Немьщкого [33, с. 39] имеем $J_{n} \rightarrow+0$ при $n \rightarrow+\infty$. Таким образом, выполнены все условия теоремы 4.1.

В заключение мы хотим выразить искреннюю признательность В. М. Миллионшикову за весьма ценные замечания, улучшившие содержание работы.

\section{Список литературы}

1. Корпусов М. О., Свешников А.Г. Трехмерные нелинейные эволюционные уравнения псевдопараболического типа в задачах математической физики // ЖВМиМФ. 2003. T. 43. № 12 . C. $1835-1869$.

2. Корпусов М. О., Свешников А.Г. Трехмерные нелинейные эволюционные уравнения псевдопараболического типа в задачах математической физики. Ч. 2 // ЖВМиМФ. 2004. T. 44. №11. C. 2041-2048.

3. Соболев С. Л. Об одной новой задаче математической физики // Изв. АН СССР. Сер. матем. 1954. Т. 18. № 1 . С. 3-50.

4. Габов C.A., Свешников А.Г. Линейные задачи теории нестационарных внутренних волн. М.: Наука, 1990. 
5. Демиденко Г. В., Успенский С. В. Уравнения и системы, не разрешенные относительно старшей производной. Новосибирск: Научная книга, 1998.

6. Плетнер Ю. Д. Фундаментальные решения операторов типа Соболева и некоторые начально-краевые задачи // ЖВМиМФ. 1992. Т. 32. №12. С. 1885-1899.

7. Свиридюк Г. А. К общей теории полугрупп операторов // УМН. 1994. Т. 49. № 4. C. $47-74$.

8. Levine H. A. Some nonexistence and instability theorems for solutions of formally parabolic equations of the form $P u_{t}=-A u+F(u) / /$ Arch. Rational. Mech. Analys. 1973. V. 51. P. $371-386$.

9. Levine H.A., Park S.R., Serrin J. Global existence and nonexistence theorems for qusilinear evolution equations of formally parabolic type // J. Diff. equat. 1998. V. 142. P. 212-229.

10. Кожанов А. И. Начально-краевая задача для уравнений типа обобщенного уравнения Буссинеска с нелинейным источником // Матем. заметки. 1999. Т. 65. № 1. С. 70-75.

11. Корпусов М. О. О разрушении решений класса сильно нелинейных уравнений типа Соболева // Изв. РАН. Сер. матем. 2004. Т. 68. № 4. С. 151-204.

12. Корпусов М. О., Свешников А.Г. Разрушение решений абстрактных задач Коши для нелинейных дифференциально-операторных уравнений // Докл. РАН. 2005. Т. 401. № 1. C. $1-3$.

13. Корпусов М.О., Свешников А.Г. О разрушении решений одного класса квазилинейных волновых диссипативных уравнений псевдопараболического типа с источниками // Докл. РАН. 2005 (в печати).

14. Митидиери Э., Похожаев С. И. Априорные оценки и отсутствие решений дифференциальных неравенств в частных производных // Тр. МИАН. 2001. Т. 229. С. 1-232.

15. Шишмарёв И. А. Об одном нелинейном уравнении типа Соболева // Дифф. уравн. 2005. T. 41. № 1. C. 1-3.

16. Karch G. Asymptotic behavior of solutions to some pseudoparabolic equations // Math. Methods Appl. Sci. 1997. V. 20. № 3. P. 271-289.

17. Goldstein J. A., Kajikiya R., Oharu S. On some nonlinear dispersive equations in several space variables // Diff. and Integ. Equat. 1990. V. 3. № 4. P. 617-632.

18. Zhang L. Decay of solutions of generalized BBMB equations in n-space dimensions // Nonlinear Anal. TMA. 1995. V. 20. P. 1343-1390.

19. Naumkin P.I. Large-time asymptotic behaviour of a step for the Benjamin-BonaMahony-Burgers equation // Proc. R. Soc. Edinb. Sect. A. 1996. V. 126. № 1. P. 1-18.

20. Avrin J., Goldstein J.A. Global existence for the Benjamin-Bona-Mahony equation in arbitrary dimensions // Nonlinear Anal. TMA. 1985. V. 9. № 8. P. 861-865.

21. Albert J. P. On the decay of solutions of the generalized Benjamin-Bona-Mahony equation // J. Math. Anal. Appl. 1989. V. 141. № 2. P. 527-537.

22. Lee H. Y., Ohm M.R., Shin J. Y. The convergence of fully discrete Galerkin approximations of the Rosenau equation // Korean J. Comput. Appl. Math. 1999. V. 6. № 1. P. 1-13.

23. Mei M. Long-time behavior of solution for Rosenau-Burgers equation. II // J. Appl. Analys. 1998. V. 68. № 3-4. P. 333-356.

24. Робертсон A., Робертсон В. Топологические векторные пространства. М.: Мир, 1967.

25. Скрыпник И. В. Методы исследования нелинейных эллиптических граничных задач. М.: Наука, 1990.

26. Лионс ЖК.-Л. Некоторые методы решения нелинейных краевых задач. М.: Мир, 1972.

27. Постников М. М. Лекции по геометрии. Семестр 2. Линейная алгебра. М.: Наука, 1986. 
28. Петровский И. Г. Лекции по теории обыкновенных дифференциальных уравнений. М.: Наука, 1970.

29. Демидович В. П. Лекции по математической теории устойчивости. М.: Наука, 1967.

30. Морен К. Методы гильбертова пространства. М.: Мир, 1965.

31. Дьяченко М. И., Ульянов П. Л. Мера и интеграл. М.: Факториал, 1998.

32. Успенский C. В., Демиденко Г. В., Перепелкин В. Г. Теоремы вложения и приложения к дифференциальным уравнениям. Новосибирск: Наука, 1984.

33. Красносельский M. А. Топологические методы в теории нелинейных интегральных уравнений. М.: Гостехиздат, 1956. 